\title{
SYNTAX AND DEFINING EQUATIONS FOR AN INTERRUPT MECHANISM IN PROCESS ALGEBRA
}

\author{
J.C.M. BAETEN*, J.A. BERGSTRA** and J.W. KLOP*** \\ * University of Amsterdam \\ ** University of Amsterdam, University of Utrecht \\ *** Centre for Mathematics and Computer Science, Amsterdam
}

ABSTRACT

\begin{abstract}
A mechanism is introduced to describe priorities in $A C P$, the algebra of communicating processes, whereby some actions have priority over others in a non-deterministic choice (or sum). This mechanism can be used to model the working of interrupts in a distributed system. This is illustrated in an extensive example.

1980 MATHEMATICS SUBJECT CLASSIFICATION: 68B10, 68C01, 68D25, 68F20. 1982 CR CATEGORIES: F.1.1, F.1.2, F.3.2, F.4.3.

KEY WORDS \& PHRASES: concurrency, process algebra, interrupts, priorities. NOTE: This work was sponsored in part by ESPRIT contract METEOR (nr. 432).
\end{abstract}

\section{Introduction}

Process algebra is an algebraical theory of concurrency, i.e. a theory about concurrent, communicating processes. Almost anything can constitute a process: the execution of a program on a computer, or the execution of an algorithm by a person, but also a game of chess or the behaviour of a vending machine.

The starting point for process algebra is the modular structure of concurrent processes at a given level of abstraction: we consider systems built up from certain basic processes by means of composition tools, including sequencing, alternative choice and parallel composition.

Process algebra tries to find laws or axioms for these composition operators, based on some a priori considerations of what features concurrent communicating processes should certainly have. Thus, we use the axiomatic method; after having established the axioms we can study different models of the theory, thus obtaining actual semantics. We mention some of the ways in which semantics can be obtained:

* by projective limits as in Bergstra \& Klop [4];

* by topological completion as in de Bakker \& Zucker [1];

* by bisimulations as defined in Park [10] and Milner [9].

In this paper, we will not deal with models at all.

In this introduction, we will just give an informal introduction to the composition operators used in process algebra. For a more technical introduction, we refer the reader to Bergstra \& Klop [4], where also a review of related approaches and comparisons with them can be found, or to Bergstra \& Tucker [5].

Process algebra starts from a collection of given objects, called atomic actions, atoms or steps. These actions are taken to be indivisible, usually have no duration and form the basic building blocks of our systems. The first two compositional operators we consider are $\cdot$, denoting sequential composition, and + for alternative composition. If $x$ and $y$ are two processes, then $x \cdot y$ is the process that starts the execution of $y$ after the completion of $x$, and $x+y$ is the process that chooses either $x$ or $y$ and executes the 
chosen process (not the other one). Each time a choice is made, we choose from a set of alternatives. We do not specify whether the choice is made by the process itself, or by the environment. Axioms Al-5 in table 1 below give the laws that + and $\cdot$ obey. We leave out $\cdot$ and brackets as in regular algebra, so $x y+z$ means $(x \cdot y)+z$.

On intuitive grounds $x(y+z)$ and $x y+x z$ present different mechanisms (the moment of choice is different), and therefore, an axiom $x(y+z)=x y+x z$ is not included.

We have a special atom $\delta$ denoting deadlock, the acknowledgement of a process that it cannot do anything anymore, the absence of any alternative. Axioms A6-7 give the laws for $\delta$.

Next, we have the parallel composition operator \|, called merge. The merge of processes $x$ and $y$ will interleave the actions of $x$ and $y$, except for the communication actions. In $x \| y$, we can either do a step from $x$, or a step from $y$, or $x$ and $y$ both synchronously perform an action, which together make up a new action, the communication action. This trichotomy is expressed in axiom CMI. Here, we use two auxiliary operators $\mathbb{L}$ (left-merge) and $\mid$ (communication merge). Thus, $x \llbracket y$ is $x \| y$, but with the restriction that the first step comes from $x$, and $x \mid y$ is $x \| y$ with a communication step as the first step. Axioms $C M 2-9$ and $C 1-3$ give the laws for $\mathbb{L}$ and $\mid$. Finally, we have in table 1 the encapsulation operator $\partial_{H}$. Here $H$ is a set of atoms, and $\partial_{H}$ blocks those actions, renames them into $\partial$. The operator $\partial_{H}$ can be used to encapsulate a process, i.e. to block communications with the environment.

Together, these operators make up the axiom system $A C P$, the algebra of communicating processes, in table 1. In this system, we cannot describe the working of an interrupt. However, for the development of process algebra it is essential to incorporate an interrupt mechanism in order to enhance its expressive power as a specification and verification formalism for concurrent systems.

In this document a new piece of syntax together with semantics defining equations is introduced. Based on a partial ordering on processes, $>$, an operator $\theta$ is defined. Now $\theta(x)$ is a context of $x$ inside which action $a$ has priority over $b$ whenever $a>b$. Interrupts will have higher priority than other actions. We think that the operator $\theta$ will have many other uses, besides the modelling of interrupts. To the best of our knowledge, the present formalization is new, at least within the theory stream around CCS and CSP.

$A C P$ with the operator $\theta$ added will yield the axiom system $A C P_{\theta}$. In section 2, we prove that this axiom system has nice theoretical properties. In the rest of the paper, we give several examples of the use of $\theta$. For verification purposes, we need an abstraction operator in some of the examples, and therefore we make use of the axiom system $A C P_{\tau}$, that was introduced in Bergstra \& Klop [2]. $A C P_{\tau}$ can be seen as a remodularization of the basic concepts of $C C S$ (see Milner [9]).

We must leave as an open issue whether or not $A C P_{\theta}$ and $A C P_{\tau}$ can be combined into $A C P_{\tau}$. From an intuitive point of view this is not clear as there is no reason why "abstraction" and "interrupts" should commute.

Table of contents:

1. Definitions and motivation

2. Theoretical matters

3. Simple examples

4. Example: a toy distributed system.

\section{Definitions and motivation}

1.1. Let $a, b, c$ be (atomic) actions, and suppose we want $a$ to take precedence over $b$ and $c$, we want $a$ to have priority over $b$ and $c$, which we will express by

$$
a>b \text { and } a>c
$$

(here $>$ is some partial order on atomic actions). Relative to this partial order, we want to define an operator $\theta$ that models this priority, so we want

$$
\begin{aligned}
& \text { i) } \theta(a+b)=a ; \theta(a+c)=a \text {; } \\
& \text { ii) } \theta(b+c)=b+c
\end{aligned}
$$


So $\theta$ chooses $a$ over $b$ or $c$, but $\theta$ does not choose between $b$ and $c$, since they are not $>$-comparable. We will define $\theta$ in an axiomatic way, in the framework of process algebras. Therefore, we will first review the axiom system ACP, the algebra of communicating processes (see Bergstra \& Klop [4]).

1.2. The signature of $\mathrm{ACP}$ is as follows: $A$ is a given finite set of atoms, $A \subseteq P$, the set of processes. On $P$ we have the following operations:

\begin{tabular}{|cl|}
\hline+ & alternative composition (sum) \\
- & sequential composition (product) \\
$\|$ & parallel composition (merge) \\
$\mathbb{L}$ & left-merge \\
$\mid$ & communication merge \\
$\partial_{H}$ & encapsulation \\
$\delta$ & deadlock
\end{tabular}

The first five operations are binary; $\partial_{H}$ is a unary operation for each $H \subseteq A$ and $\delta \in A$ is a constant.

1.3. The set of axioms of ACP is as follows (see Bergstra \& Klop [4]):

\begin{tabular}{|c|c|c|c|}
\hline$x+y=y+x$ & $\mathrm{Al}$ & $a|b=b| a$ & $\mathrm{Cl}$ \\
\hline$x+(y+z)=(x+y)+z$ & $\mathrm{~A} 2$ & $(a \mid b)|c=a|(b \mid c)$ & $\mathrm{C} 2$ \\
\hline$x+x=x$ & A. 3 & $\delta \mid a=\delta$ & $\mathrm{C} 3$ \\
\hline$(x+y) z=x z+y z$ & A4 & & \\
\hline$(x y) z=x(y z)$ & A5 & $\partial_{H}(a)=a \quad$ if $a \notin H$ & D1 \\
\hline$x+\delta=x$ & A6 & $\partial_{H}(a)=\delta$ if $a \in H$ & D2 \\
\hline$\delta x=\delta$ & A7 & $\partial_{H}(x+y)=\partial_{H}(x)+\partial_{H}(y)$ & D3 \\
\hline & & $\partial_{H}(x y)=\partial_{H}(x) \cdot \partial_{H}(y)$ & D4 \\
\hline$x \| y=x \amalg y+y \amalg x+x \mid y$ & CM1 & & \\
\hline$a \Perp x=a x$ & CM2 & & \\
\hline$(a x)\lfloor y=a(x \| y)$ & $\mathrm{CM} 3$ & & \\
\hline$(x+y) \llbracket z=x \amalg z+y \amalg z$ & $\mathrm{CM} 4$ & & \\
\hline$(a x) \mid b=(a \mid b) x$ & CM5 & & \\
\hline$a \mid(b x)=(a \mid b) x$ & CM6 & & \\
\hline$(a x) \mid(b y)=(a \mid b)(x \| y)$ & CM7 & & \\
\hline$(x+y)|z=x| z+y \mid z$ & CM8 & & \\
\hline$x|(y+z)=x| y+x \mid z$ & CM9 & & \\
\hline
\end{tabular}

Table 1.

Here $a, b \in A, x, y, z \in P, H \subseteq A$.

If we view these equations as rewrite rules, going from left to right, and add a rule A2': $(x+y)+z=x+(y+z)$, we get a term rewrite system RACP. We quote three theorems from Bergstra \& Klop [4]: 
Theorem. (i) RACP is confluent (has the Church-Rosser property);

(ii) RACP is strongly terminating (working modulo $\mathrm{Al}$ and $\mathrm{A} 2$ );

(iii) (elimination) for each ACP-term $s$ there is an ACP-term $t$ not containing $\|, \mathbb{L}, \mid, \partial_{H}$, such that $\mathrm{ACP}+s=t$.

1.4 Now we return to the problem in 1.1: we want to extend ACP with an operator $\theta$ and give some defining equations for it, so that (i) and (ii) of 1.1 are satisfied. So suppose we have a partial order $<$ on $A$ so that $\delta$ is minimal, i.e. we have

$$
\begin{array}{ll}
\text { 1. } & \text { not }(a<a) \\
\text { 2. } & a<b \Rightarrow \operatorname{not}(b<a) \\
\text { 3. } & a<b \& b<c \Rightarrow a<c \\
\text { 4. } & \delta<a(\text { if } a \neq \delta)
\end{array}
$$

for all $a, b, c \in A$.

In order to define $\theta$, we first need to define an auxiliary operator $\varangle: P \times P \rightarrow P$.

$$
x \triangleleft y
$$

is pronounced $\mathrm{x}$ unless $y$. In the following we will explain $\varangle$. First, on $A$ we have

$$
\begin{array}{lll}
\text { P1 } & a \triangleleft b=a & \text { if not }(a<b) \\
\text { P2 } & a \triangleleft b=\delta & \text { if } a<b
\end{array}
$$

So $a \triangleleft b$ is equal to $a$, unless $b$ has priority over $a$, in which case $a \triangleleft b$ becomes $\delta$. In general, we get $x \triangleleft y$ in the following way:

Suppose $x$ is a sum $\Sigma_{i=1}^{N} a_{i} x_{i}, a_{i} \in A, x_{i} \in P$. Then, in $x$ we throw away (i.e. set equal to $\delta$ ) those branches $a_{i} x_{i}$ such that there is a branch $b_{j} y_{j}$ in $y$ with $a_{i}<b_{j}$; other branches we leave untouched.

1.5 Example Suppose $A=\{\delta, a, b, c, d\}$ and we have the following p.o. on A:

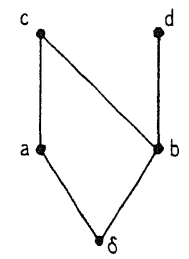

(In this picture, we have e.g. $b<c$ because $c$ is located above $b$ and there is a line connecting them; so not $(a<d)$.) We do some calculations: $(x, y, z \in P)$.
a) $\quad(a x+b y+c) \triangleleft d z=(a x+b y+c) \triangleleft d=a x+\delta+c=a x+c$
b) $\quad(a x+b y+c) \triangleleft c=\delta+\delta+c=c$
c) $x \triangleleft \delta=x$

1.6 We now present the axiom system $\mathrm{ACP}_{\theta}$ (see table 2). 


\begin{tabular}{|c|c|c|c|}
\hline$x+y=y+x$ & $\mathrm{Al}$ & $a \triangleleft b=a$ if not $(a<b)$ & $\mathrm{Pl}$ \\
\hline$x+(y+z)=(x+y)+z$ & $\mathrm{~A} 2$ & $a \triangleleft b=\delta$ if $a<b$ & $\mathrm{P} 2$ \\
\hline$x+x=x$ & A3 & $x \triangleleft y z=x \triangleleft y$ & P3 \\
\hline$(x+y) z=x z+y z$ & A4 & $x \triangleleft(y+z)=(x \triangleleft y) \triangleleft z$ & P4 \\
\hline$(x y) z=x(y z)$ & A5 & $x y \triangleleft z=(x \triangleleft z) y$ & P5 \\
\hline$x+\delta=x$ & A6 & $(x+y) \triangleleft z=x \triangleleft z+y \triangleleft z$ & P6 \\
\hline$\delta x=\delta$ & A7 & & \\
\hline$a|b=b| a$ & $\mathrm{Cl}$ & & \\
\hline$(a \mid b)|c=a|(b \mid c)$ & $\mathrm{C} 2$ & & \\
\hline$\delta \mid a=\delta$ & $\mathrm{C} 3$ & & \\
\hline$x \| y=x \Perp y+y \amalg x+x \mid y$ & CMl & $\theta(a)=a$ & $\mathrm{TH} 1$ \\
\hline$a \llbracket x=a x$ & $\mathrm{CM} 2$ & $\theta(x y)=\theta(x) \cdot \theta(y)$ & $\mathrm{TH} 2$ \\
\hline$a x \mathbb{L} y=a(x \| y)$ & $\mathrm{CM} 3$ & $\theta(x+y)=\theta(x) \triangleleft y+\theta(y) \triangleleft x$ & TH3 \\
\hline$(x+y) \llbracket z=x \llbracket z+y \llbracket z$ & CM4 & & \\
\hline$(a x) \mid b=(a \mid b) x$ & CM5 & & \\
\hline$a \mid(b x)=(a \mid b) x$ & CM6 & & \\
\hline$(a x) \mid(b y)=(a \mid b)(x \| y)$ & CM7 & & \\
\hline$(x+y)|z=x| z+y \mid z$ & $\mathrm{CM} 8$ & & \\
\hline$x|(y+z)=x| y+x \mid z$ & $\mathrm{CM} 9$ & & \\
\hline$\partial_{H}(a)=a$ if $a \notin H$ & D1 & & \\
\hline$\partial_{H}(a)=\delta$ if $a \in H$ & D2 & & \\
\hline$\partial_{H}(x+y)=\partial_{H}(x)+\partial_{H}(y)$ & D3 & & \\
\hline$\partial_{H}(x y)=\partial_{H}(x) \cdot \partial_{H}(y)$ & D4 & & \\
\hline
\end{tabular}

1.7 Let us verify (i) and (ii) of 1.1 and some other formulas: $(b<a$ and $c<a$ )

i) $\quad \theta(a+b)=\theta(a) \triangleleft b+\theta(b) \triangleleft a=a \triangleleft b+b \triangleleft a=a+\delta=a$.

ii) $\quad \theta(b+c)=\theta(b) \triangleleft c+\theta(c) \triangleleft b=b \triangleleft c+c \triangleleft b=b+c$.

iii) $\quad \theta(b(a+c))=\theta(b) \cdot \theta(a+c)=b(\theta(a) \triangleleft c+\theta(c) \triangleleft a)=b(a \triangleleft c+c \triangleleft a)=b(a+\delta)=b a$.

iv) $\quad \theta(a+b+c)=\theta(a) \triangleleft(b+c)+\theta(b+c) \triangleleft a=(\theta(a) \triangleleft b) \triangleleft c+(\theta(b) \triangleleft c+\theta(c) \triangleleft b) \triangleleft a=$ $(a \triangleleft b) \triangleleft c+(b \triangleleft c+c \triangleleft b) \triangleleft a=a \triangleleft c+(b+c) \triangleleft a=a+\delta=a$.

v) $\quad \theta(a+b+c)=\theta(a+b) \triangleleft c+\theta(c) \triangleleft(a+b)=(\theta(a) \triangleleft b+\theta(b) \triangleleft a) \triangleleft c+(\theta(c) \triangleleft a) \triangleleft b=$ $(a \triangleleft b+b \triangleleft a) \triangleleft c+(c \triangleleft a) \triangleleft b=(a+\delta) \triangleleft c+\delta \triangleleft b=a \triangleleft c+\delta \triangleleft c+\delta=a+\delta+\delta=a$.

1.8 In section 2, we will prove that this axiom system is well-behaved. Among other things, we prove a theorem analogous to 1.3 for $\mathrm{ACP}_{\theta}$.

1.9 Note: about leaving out parentheses: we take $\cdot$ to be more binding then other operations and + to be less binding than other operations, so we write $x y \triangleleft z$ for $(x y) \triangleleft z$ and $x+y \triangleleft z$ for $x+(y \triangleleft z)$.

\section{Theoretical matters}

We will write $\mathrm{ACP}_{\theta}$ as a term rewrite system, and prove confluency and termination. We find that $\mathrm{ACP}_{\theta}$ is a conservative extension of $\mathrm{ACP}$, and prove an elimination theorem. 
2.1 Lemma The following identities hold in $\mathrm{ACP}_{\theta}$ :

$$
\begin{array}{ll}
\text { P7 } & (x \triangleleft y) \triangleleft z=(x \triangleleft z) \triangleleft y \\
\text { P8 } & (x \triangleleft y) \triangleleft y=x \triangleleft y \\
\text { TH4 } & \theta(x) \triangleleft x=\theta(x)
\end{array}
$$

Proof. Above the equality signs, we indicate which rule is being used. Let $x, y, z \in P$.

P7: $(x \triangleleft y) \triangleleft z \stackrel{P 4}{=} x \triangleleft(y+z) \stackrel{A 1}{=} x \triangleleft(z+y) \stackrel{P 4}{=}(x \triangleleft z) \triangleleft y$

P8: $(x \triangleleft y) \triangleleft y \stackrel{P 4}{=} x \triangleleft(y+y) \stackrel{A 3}{=} x \triangleleft y$

TH4: $\theta(x) \triangleleft x \stackrel{A 3}{=} \theta(x) \triangleleft x+\theta(x) \triangleleft x \stackrel{T H 3}{=} \theta(x+x) \stackrel{A 3}{=} \theta(x)$.

2.2 Definition: We define the term rewrite system $\mathrm{RACP}_{\theta}$ as follows: take the term rewrite system RACP (see 1.3, rules $\left.\mathrm{Al} 1,2,2^{\prime}, 3-7, \mathrm{Cl}-3, \mathrm{CM} 1-9, \mathrm{D} 1-4\right)$, and add rules $\left\{\begin{array}{l}\mathrm{P} 1-8 \\ \mathrm{TH} 1-4\end{array}\right.$.

(Reading them as reductions, from left to right)

Note: when proving termination, we will have to work modulo rules A1 A2, A2', P7, since applying Al or P7 twice gives back the original term, and A2' undoes the effect of A2.

We can now state our main theorem:

\subsection{Theorem}

i) $\mathrm{RACP}_{\theta}$ is confluent (has the Church-Rosser property);

ii) $\mathrm{RACP}_{\theta}$ is strongly terminating (working modulo $\mathrm{A} 1, \mathrm{~A} 2, \mathrm{P} 7$ );

iii) (elimination) for each $\mathrm{ACP}_{\theta}$-term $s$ there is a term $t$ not containing $\varangle, \theta, \|, \mathbb{L}, \mid, \partial_{H}$ such that $\mathrm{ACP}_{\theta}+s=t$

iv) $\mathrm{ACP}_{\theta}$ is a conservative extension of $\mathrm{ACP}$, i.e. for all $\mathrm{ACP}$-terms $s, t$ we have:

$$
\mathrm{ACP}+s=t \Leftrightarrow \mathrm{ACP}_{\theta}+s=t .
$$

Strategy of proof: we first prove (ii) in 2.4-2.12 and then (i) in 2.13-2.15. The elimination (iii) then follows immediately from (ii) and (iv) follows if we combine (i) and (iii).

2.4 To prove (ii), we use the method of recursive path orderings of Dershowitz (see Dershowitz [6] and Bergstra \& Klop [2]). The idea of this method is, that we assign a certain kind of tree to each term in $\mathrm{ACP}_{\theta}$. We have a partial ordering $\Rightarrow$ on these trees, and it follows from the Kruskal Tree Theorem (see Dershowitz [6]) that this ordering is well-founded. Then, if we show that for each reduction in $\mathrm{ACP}_{\theta}$, the trees of the terms before and after the reduction are ordered by $\Rightarrow$, we have shown that each reduction in $\mathrm{ACP}_{\theta}$ must terminate.

2.5 Definition: Let $D$ be the set of all finite commutative rooted trees whose nodes are labeled with natural numbers.

Example:

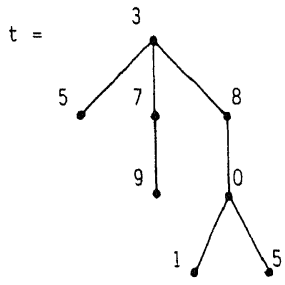


Notation: $t=3(5,7(9), 8(0,(1,5)))=3(8(0(1,5)), 5,7(9))$.

Definition: We define a partial order $\Rightarrow$ on $D$ as follows: $t=n\left(t_{1}, \ldots, t_{k}\right) \Rightarrow m\left(s_{1}, \ldots, s_{l}\right)=s$ $(k \geqslant 0, l \geqslant 0)$ iff

(i) $n>m$ and $t \Rightarrow s_{i}$ for all $i=1, \ldots, l$; or

(ii) $n=m$ and for each $i \leqslant l$ there is a different $j \leqslant k$ such that $t_{j} \Rightarrow s_{i}$ : also, for at least one $i \leqslant l$ we have $t_{j} \Rightarrow s_{i}$. (so $t_{j} \neq s_{i}$ ); or

(iii) $n<m$ and $t_{i} \Rightarrow s$ for some $j \leqslant k$.

2.6 Theorem (Dershowitz [6]) $\Rightarrow$ is a well-founded partial order on $D$.

2.7 Let $s, t$ be $\mathrm{ACP}_{\theta}$-terms, and let $s \stackrel{r}{\rightarrow} t$ symbolize that $s$ reduces to $t$ in a one-step, outermost reduction by rule $r$. Now we want to define a mapping $\phi$ from terms of $\mathrm{ACP}_{\theta}$ to elements of $D$ such that the following hold:

i) if $s \stackrel{r}{\rightarrow} t$ and $r=\mathrm{Al}, \mathrm{A} 2, \mathrm{~A} 2$, $\mathrm{P} 7$, then $\phi(s)=\phi(t)$;

ii) if $s \stackrel{r}{\rightarrow} t$ and $r$ is any other rule, then $\phi(s) \Rightarrow \phi(t)$. By Dershowitz' theorem, this suffices to show 2.3.ii, the termination of $\mathrm{RACP}_{\theta}$.

2.8 Before we can define $\phi$, we need two more definitions.

Definition: Let $u$ be an $\mathrm{ACP}_{\theta}$-term. We define st $(u)$, the standard part of $u$ or ACP-part of $u$, inductively.

i) $s t(u)=u$ if $u$ is an ACP-term;

ii) if $u \equiv x \square y,(\square=+,,, \|, \mathbb{L}, \mid)$, then $s t(u)=s t(x) \square$ st $(y)$;

iii) if $u \equiv \partial_{H}(x)$, then $s t(u)=\partial_{H}(s t(x))$;

iv) if $u \equiv x \triangleleft y$, then $s t(u)=s t(x)$;

v) if $u \equiv \theta(x)$, then $s t(u)=\operatorname{st}(x)$.

So we obtain st $(u)$ by leaving out all right hand sides $y$ of subterms $x \varangle y$, and then leaving out all $\triangleleft$ and $\theta$. It is obvious that $s t(u)$ is an ACP-term.

Definition: Let $u$ be an $\mathrm{ACP}_{\theta}$-term. We define $|u|$, the norm of $u$, by

$$
|u|=\# G(s t(u))
$$

so $|u|$ is the number of symbols in the reduction graph $\mathcal{S}$ of $s t(u)$. Note that an immediate consequence of this definition is that for all $\mathrm{ACP}_{\theta}$-terms $x, y$ :

a) $|x|+|y|<|x+y|$

b) $|x|+|y|<|x \cdot y|$.

2.9 Definition: We define $\phi$ inductively. Let $t$ be an $A C P_{\theta^{-}}$term

i) $t \equiv a \in A . \phi(a)=.1$ (a single node labeled by 1 )

ii) $t \equiv \partial_{H}(x)$. Then $\phi(t)=$

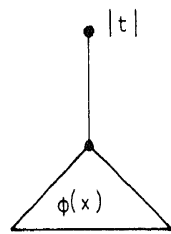


iii) $t \equiv x \square y$, with $\square=+,, \|, \mathbb{L}$ or $\mid$

Then $\phi(t)=$

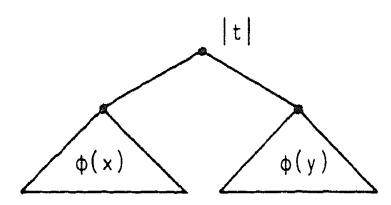

fig.4

iv) $t \equiv x \triangleleft y$. Here we have an intermediate step. First we rewrite $\mathrm{x}$ as $\left(\cdots\left(\left(z \triangleleft y_{1}\right) \triangleleft y_{2}\right) \triangleleft \cdots \triangleleft y_{k}\right)$ $(k \geqslant 0)$, so that the main connective in $z$ is not $\varangle$ (in case $k=0$, we have $z \equiv x$ ). We use the notation $x=z \triangleleft\left\{y_{1}, \ldots, y_{k}\right\}$, so $t=z \triangleleft\left\{y, y_{1}, \ldots, y_{k}\right\}$, where on the right-hand side of the $\triangleleft$ we have a multiset. Axiom P7 gives us the justification for doing this. Then we define $\phi(t)=$

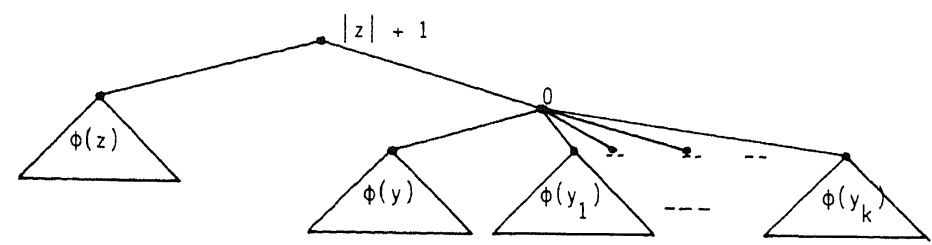

fig. 5

If $\sigma=\left\{y_{1}, \ldots, y_{k}\right\}$, we use the abbreviation

$\phi(t)=$

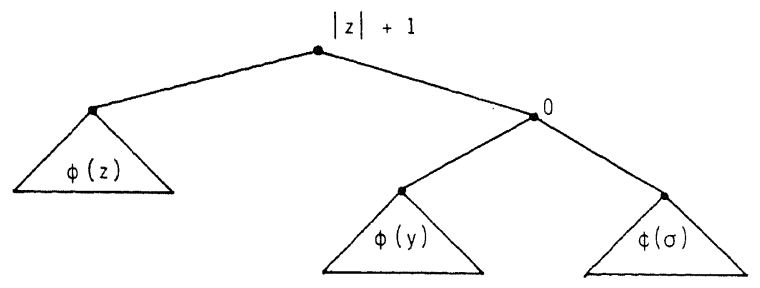

fig.6

v) $t \equiv \theta(x)$. Put $\phi(t)=$

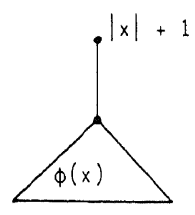

fig.7 
2.10 Examples: Let $a, b, c \in A$, then
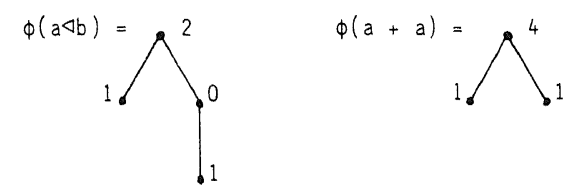

fig. $8 \mathrm{a}, \mathrm{b}, \mathrm{c}$

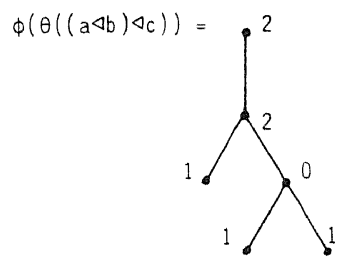

2.11 Now we will prove claims (i) and (ii) of 2.6.

So suppose we have $t \stackrel{r}{\rightarrow} t^{\prime}$, redex $t$ reduces to $t^{\prime}$ (outermost) by an application of rule $r$. We shall consider 3 cases.

2.11.1 case 1: $r=\mathrm{A} 1, \mathrm{~A} 2, \mathrm{~A} 2$ ', P7. Since our trees are commutative, this follows from the definition of $\phi$. So for P7, $t \equiv(x \triangleleft y) \triangleleft z$ and

$\phi(t)=$

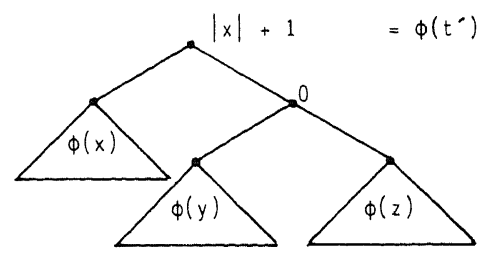

fig.9

2.11.2 case 2: $r=\mathrm{A} 3-7, \mathrm{Cl}-3, \mathrm{CM} 1-9$ or D1-4. Then $t \equiv x \square y$, for $\square=+,, \|, \mathbb{L}$ or $\mid$, or $t \equiv \partial_{H}(x)$. We also have $s t(t) \stackrel{r}{\rightarrow} s t\left(t^{\prime}\right)$, since non-standard parts cannot be instrumental in the reduction. But then, by theorem 1.3, we have $|t|>\left|t^{\prime}\right|$ (also see note 2.8). Therefore, the top of tree $\phi(t)$ has a higher number as label than the top of tree $\phi\left(t^{\prime}\right)$. It is shown in Bergstra \& Klop [2] that we must also have $\phi(t) \Rightarrow \phi\left(t^{\prime}\right)$.

2.11.3 case 3: otherwise. Because of definition 2.9.iii, we will have to look at redices $x \triangleleft \sigma$, with a finite multiset of terms on the righthandside, and $\mathrm{x}$ not of the form $(y \triangleleft z)$. Note that $x \triangleleft \varnothing \equiv x$ and this identification is correct because

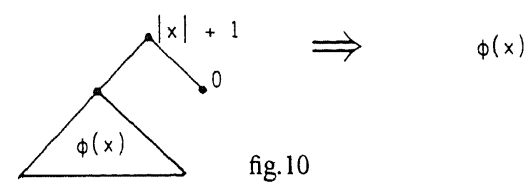

(by 2.5.iii)

We will look at each rule in turn

P1. not $(a<b) . \phi(a \triangleleft\{b\} \cup \sigma)=$

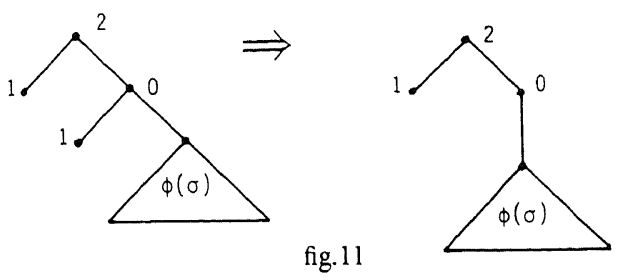


$=\phi(a \triangleleft \sigma)$.

P2. $(a<b) . \phi(a \triangleleft\{b\} \cup \sigma)=$

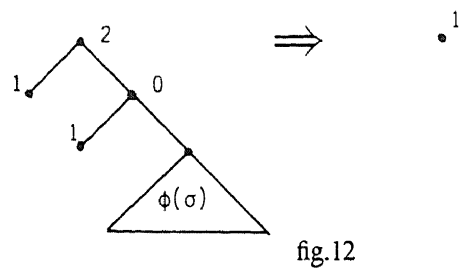

$=\phi(\delta)$

(we combine this with P8 to get $\delta \triangleleft \sigma=\delta$ )

P3. $\phi(x \triangleleft\{y z\} \cup \sigma)=$

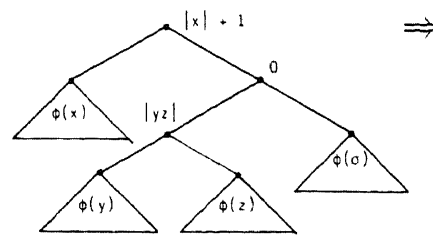

$\Longrightarrow$

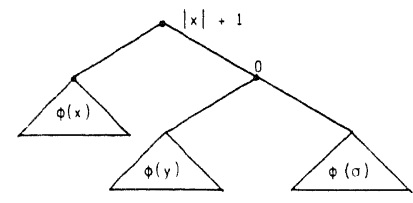

fig. 13

$=\phi(x \triangleleft\{y\} \cup \sigma)$

P4. $\phi(x \triangleleft\{y+z\} \cup \sigma)=$
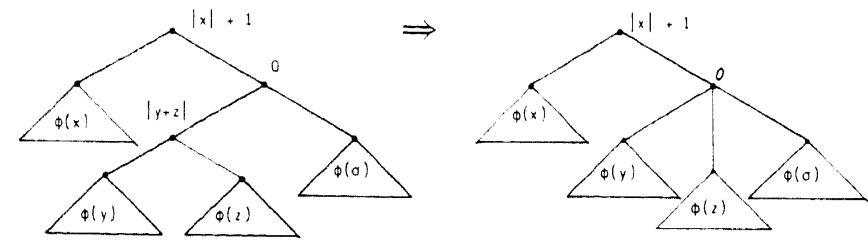

fig. 14

$=\phi(x \triangleleft\{y, z\} \cup \sigma)$

P5. $\phi(x y<\sigma)=$
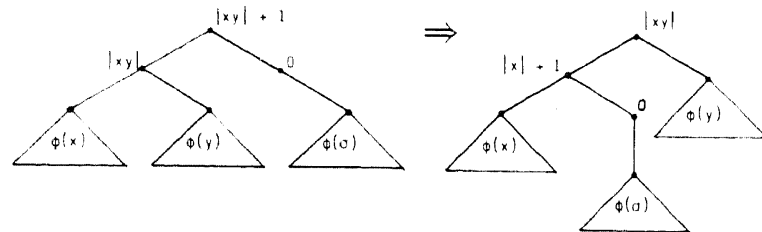

fig. 15

$=\phi((x \triangleleft \sigma) y)$

(for the proof, use 2.5.i: we have $|x y|+1>|x y|$, so we only need that the entire tree on the left majorizes both subtrees on the right)

P6. $\phi((x+y) \triangleleft \sigma)=$ 


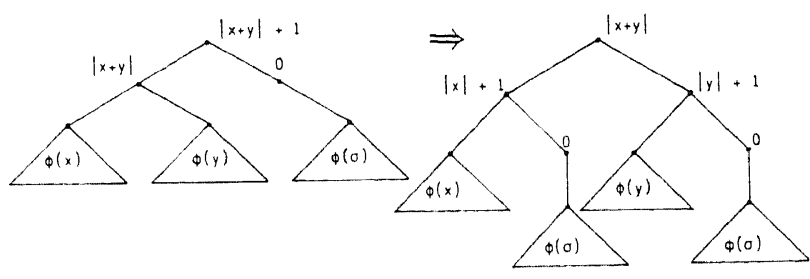

fig. 16

$=\phi(x \triangleleft \sigma+y \varangle \sigma)$. (again use 2.5.i)

P8. $\phi\left(\left(x \triangleleft\{y\} \cup \sigma_{1}\right) \triangleleft\{y\} \cup \sigma_{2}\right)=\phi(x \triangleleft\{y, y\} \cup \sigma)=$ (write $\left.\sigma=\sigma_{1} \cup \sigma_{2}\right)$

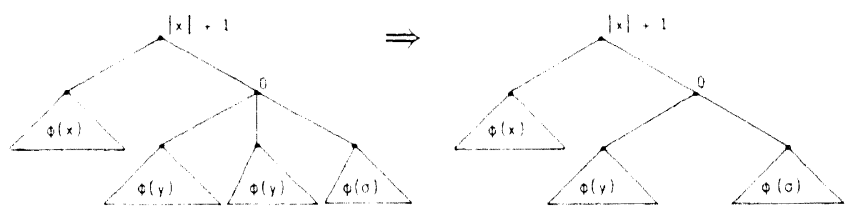

fig. 17

$=\phi(x \triangleleft\{y\} \cup \sigma)$ (by 2.5.ii)

TH1. $\phi(\theta(a))=\Gamma_{1}^{2} \Rightarrow .1=\phi(a)$.

TH2. $\phi(\theta(x y))=$

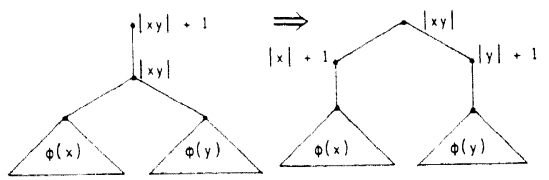

fig. 18

$=\phi(\theta(x) \cdot \theta(y))$ (use 2.5.i)

TH3. $\phi(\theta(x+y))=$

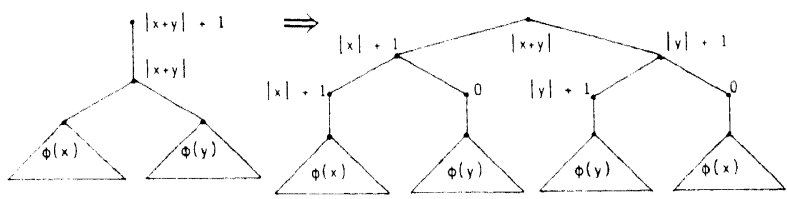

fig. 19

$=\phi(\theta(x) \triangleleft y+\theta(y) \triangleleft x)$

(again by 2.5.i). Note that in this last case we should write $x \triangleleft y=z \triangleleft\{y\} \cup \sigma$ and $y \triangleleft x=w \triangleleft\{x\} \cup \tau$, for some $z$ and $w$ not having $\triangleleft$ as the main connective. But then $|x| \geqslant|z|$, $|y| \geqslant|w|$, and the proof of $\Rightarrow$ is not harder.

TH4. $\phi(\theta(x) \triangleleft\{x\} \cup \sigma)=$ 


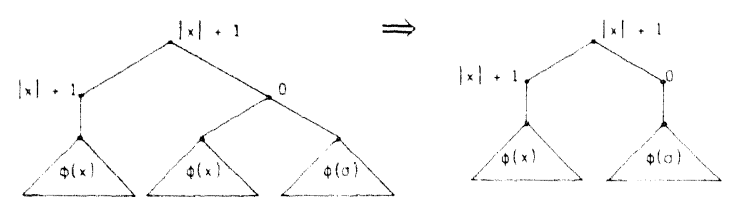

fig. 20

$=\phi(\theta(x) \triangleleft \sigma)($ by 2.5.ii).

2.12 This completes the proof of 2.3.ii: $\mathrm{RACP}_{\theta}$ is strongly terminating, working modulo $\mathrm{A} 1, \mathrm{~A} 2, \mathrm{~A} 2, \mathrm{P} 7$.

2.13 Now we turn our attention to proving 2.3.i. Since we know that $\mathrm{RACP}_{\theta}$ is strongly terminating, it is enough to show that it is weakly confluent (this follows from Newman's lemma, see Klop [8] or Huet [7]). Showing the weak confluency amounts to looking at all critical pairs (a critical pair is a redex to which two different rules can be applied) and showing that after these reductions a common reduct can be found. In other words: if $t \stackrel{r_{1}}{\rightarrow} t_{1}$ and $t \stackrel{r_{2}}{\rightarrow} t_{2}$, then there is a term $t^{\prime}$ such that both $t_{1}$ and $t_{2}$ reduce to $t^{\prime}$ (possibly in more than one step). See figure 21 .

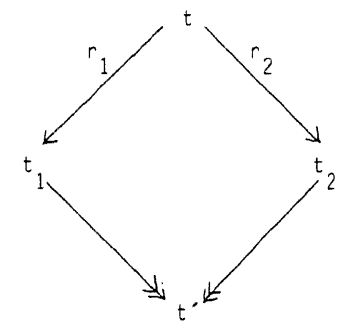

fig. 21

We will actually prove more, namely that the open theory of $\mathrm{RACP}_{\theta}$ is confluent, after adding the extra rules below (by open theory we mean the theory of finite terms, possibly containing variables). The extra rules are:

\begin{tabular}{ll|}
\hline P9 & $x \triangleleft \delta=x$ \\
P10 & $\delta \triangleleft x=\delta$ \\
C4 & $\delta \mid x=\delta$ \\
C5 & $x \mid \delta=\delta$ \\
\hline
\end{tabular}

These rules can be proved very easily for closed finite terms $\mathrm{x}$ by induction.

We know already that RACP is confluent (by 1.3), so we only have to check critical pairs involving a new rule (P1-10, TH1-4). Furthermore, Cl-5, CM1-9 and Dl-4 cannot clash with a new rule.

Still, a tedious job remains. We do the work in 2.14, in cases 2.14.1 to 2.14.46. 
2.14 Matrix of critical pairs. * means a proof is required; 0 means redices cannot overlap.

TH TH TH TH

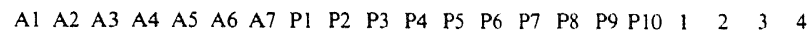

$\begin{array}{llllllllllllllllllllll}\mathrm{P} 1 & 0 & 0 & 0 & 0 & 0 & 0 & 0 & 0 & 0 & 0 & 0 & 0 & 0 & * & * & * & 0 & 0 & 0 & 0\end{array}$

$\begin{array}{lllllllllllllllllllll}\mathrm{P} 2 & 0 & 0 & 0 & 0 & 0 & 0 & 0 & 0 & 0 & 0 & 0 & 0 & * & * & * & 0 & 0 & 0 & 0\end{array}$

P3 $0 \begin{array}{lllllllllllllllllll} & 0 & 0 & * & * & 0 & * & 0 & 0 & 0 & * & * & 0 & * & 0 & 0 & 0 & *\end{array}$

P4 $\quad * \quad * \quad * \quad \begin{array}{lllllllllllllll} & * & * & * & 0 & 0 & * & * & 0 & * & 0 & 0 & 0 & *\end{array}$

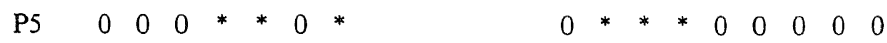

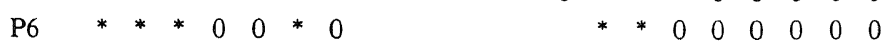

P7 $\quad 0 \begin{array}{lllllllllllllll} & 0 & 0 & 0 & 0 & 0 & 0 & 0 & 0 & 0 & 0 & 0 & 0 & 0\end{array}$

P8 $0 \begin{array}{llllllllllllll} & 0 & 0 & 0 & 0 & 0 & 0 & 0 & * & * & 0 & 0 & 0 & 0\end{array}$

P9 $\quad 0 \begin{array}{lllllllllllll} & 0 & 0 & 0 & 0 & 0 & 0 & 0 & 0 & 0 & *\end{array}$

$\begin{array}{llllllllllllll}\mathrm{P} 10 & 0 & 0 & 0 & 0 & 0 & 0 & 0 & 0 & 0 & 0 & 0\end{array}$

TH1 00000000000

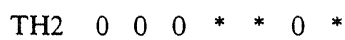

$\mathrm{TH} 3 * * * * * * \quad 0 \quad 0 \quad * \quad 0$

TH4 $00 \begin{array}{llllll}0 & 0 & 0 & 0 & 0 & 0\end{array}$

$0 \quad 0 *$

$0 *$

1. P1 \& P8. Suppose not $(a<b)$

$(a \triangleleft b) \triangleleft b \frac{\mathrm{Pl}}{\mathrm{P} 8} \overrightarrow{\mathrm{a}} \triangleleft b$.

2. P1 \& P9. For all $a \in A$, we have not $(a<\delta)$ (by $1.4 .1,2,4)$.

$a \triangleleft \delta \stackrel{\mathrm{Pl}}{\mathrm{P} 9} a$.

3. P1 \& P10. Note that not $(\delta<a)$ implies $a=\delta$. (by $1.4 .1,2,4$ )

$\delta \triangleleft \delta \stackrel{\mathrm{Pl}}{\mathrm{PlO}} \delta$.

4. $\mathrm{P} 2 \& \mathrm{P} 8$. Suppose $a<b$.

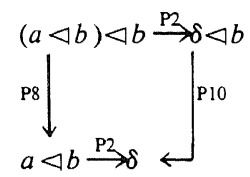

5. P2 \& P9. We can never have $a<\delta$.

6. $\mathrm{P} 2 \& \mathrm{P} 10$. Let $\delta<a . \delta \triangleleft a \frac{\mathrm{P} 2}{\mathrm{P} 10} \delta$

7. P3 \& A4.

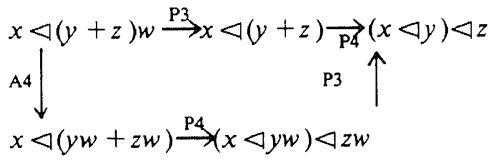


8. P3 \& A5.

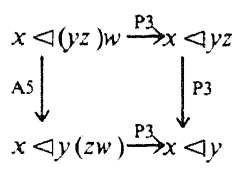

9. $\quad$ P3 \& A7. $x \triangleleft \delta y \stackrel{\mathrm{P} 3}{\mathrm{~A} 7} x \triangleleft \delta$.

10. P3 \& P7.

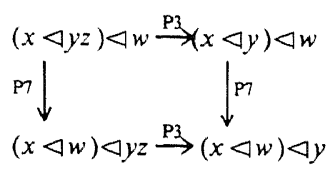

11. P3 \& P8.

$(x \triangleleft y z) \triangleleft y z \stackrel{\mathrm{P} 3 \mathrm{P} 3}{\rightarrow}(x \triangleleft y) \triangleleft y$

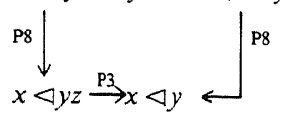

12. $\mathrm{P} 3 \& \mathrm{P} 10$.

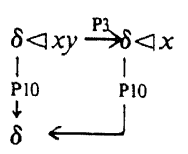

13. P3 \& TH4.

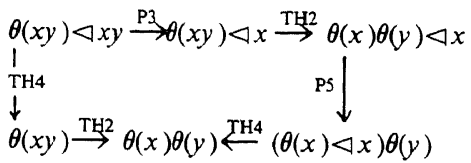

14. $\mathrm{P} 4 \& \mathrm{Al}$.

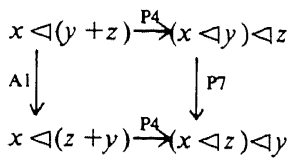

15. P4 \& A2.

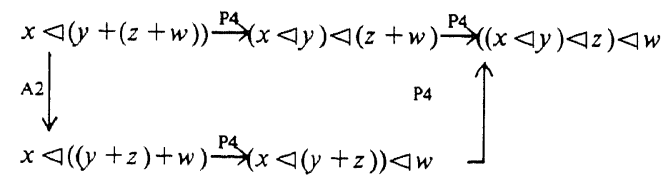

16. P4 \& A3.

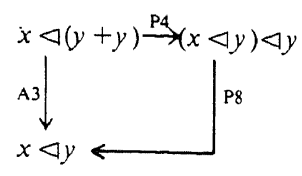


17. $\mathrm{P} 4 \& \mathrm{~A} 6$.

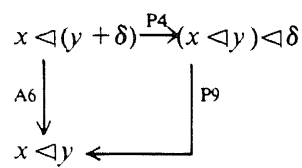

18. P4 \& P7.

$(x \triangleleft(y+z)) \triangleleft w \stackrel{\mathrm{P} 4}{\rightarrow}((x \triangleleft y) \triangleleft z) \triangleleft w$

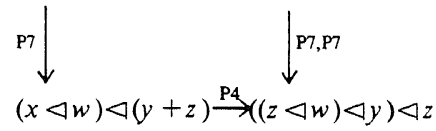

19. $\mathrm{P} 4 \& \mathrm{P} 8$.

$(x \triangleleft(y+z)) \triangleleft(y+z) \stackrel{\mathrm{P} 4 \mathrm{P} 4}{\rightarrow}(((x \triangleleft y) \triangleleft z) \triangleleft y) \triangleleft z$

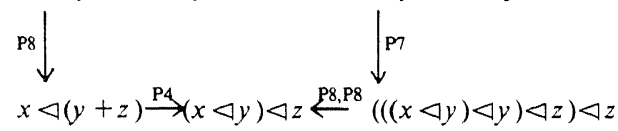

20. P4 \& P10.

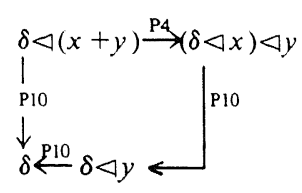

21. $\mathrm{P} 4 \& \mathrm{TH} 4$.

$\theta(x+y) \triangleleft(x+y) \stackrel{\mathrm{P} 4}{\rightarrow}(\theta(x+y) \triangleleft x) \triangleleft y \stackrel{\mathrm{IH} 3}{\rightarrow}((\theta(x) \triangleleft y+\theta(y) \triangleleft x) \triangleleft x) \triangleleft y$

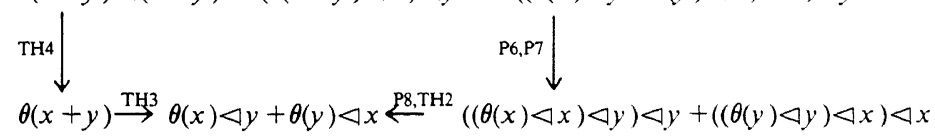

22. P5 \& A4.

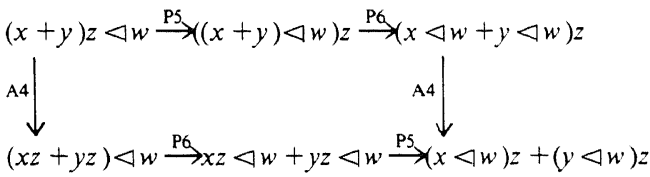

23. $\mathrm{P} 5 \& \mathrm{~A} 5$.

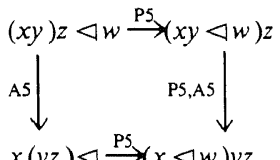

$x(y z) \triangleleft \stackrel{\mathrm{PS}}{\rightarrow}(x \triangleleft w) y z$ 
24. P5 \& A7.

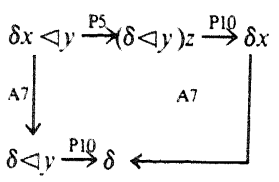

25. P5 \& P7.

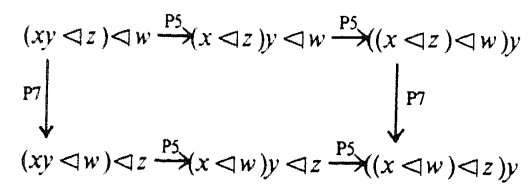

26. P5 \& P8

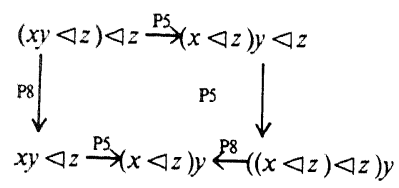

27. $\mathrm{P} 5 \& \mathrm{P} 9$.

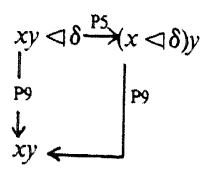

28. $\mathrm{P} 6 \& \mathrm{Al}$.

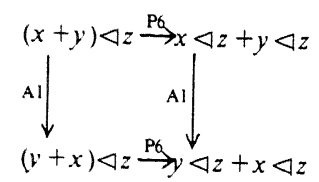

29. $\quad$ P6 \& A2.

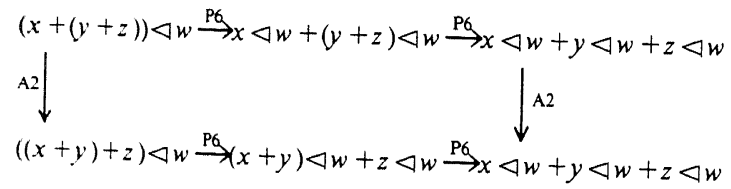

30

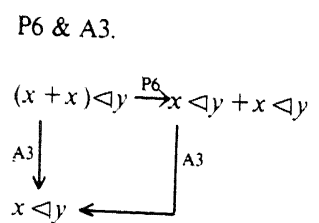


31. P6 \& A6.

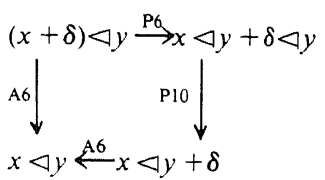

32. $\mathrm{P} 6 \& \mathrm{P} 7$.

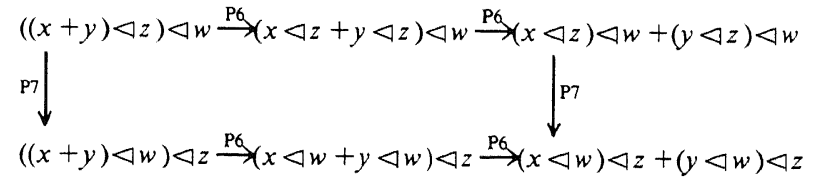

33. $\quad$ P6 \& P8.

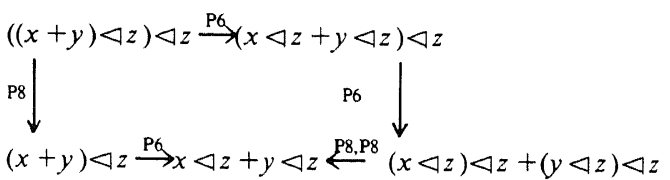

34. $\quad$ P8 \& P9.

$(x \triangleleft \delta) \triangleleft \delta \frac{\mathrm{P} q}{\mathrm{Pg}} x \triangleleft \delta$

35. $\mathrm{P} 8 \& \mathrm{P} 10$

$(\delta \triangleleft x) \triangleleft x \underset{\mathrm{P} 10}{\mathrm{P}} \delta \triangleleft x$

36. $\mathrm{P} 9 \& \mathrm{TH} 4$.

$\theta(\delta) \triangleleft \delta \frac{\mathrm{P} 9}{\mathrm{TH} 4} \theta(\delta)$

37. $\mathrm{TH} 1 \& \mathrm{TH} 4$.

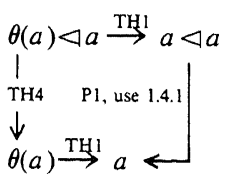

38. $\mathrm{TH} 2 \& \mathrm{~A} 4$

$\theta((x+y) z) \stackrel{\mathrm{TH} 2}{\longrightarrow} \theta(x+y) \theta(z) \stackrel{\mathrm{TH} 3}{\rightarrow}(\theta(x) \triangleleft y+\theta(y) \triangleleft x) \theta(z) \stackrel{\mathrm{A} 4}{\rightarrow} \theta(z) \triangleleft y) \theta(z)+(\theta(y) \triangleleft x) \theta(z)$

A4 $\downarrow$

$\theta(x z+y z) \stackrel{\mathrm{TH} 3}{\rightarrow} \theta(x z) \varangle y z+\theta(y z) \triangleleft x z \stackrel{\mathrm{P} 3}{\rightarrow}^{\mathrm{TH} 2} \theta(x) \theta(z) \triangleleft y+\theta(y) \theta(z)<x$

39. TH2 \& A5.

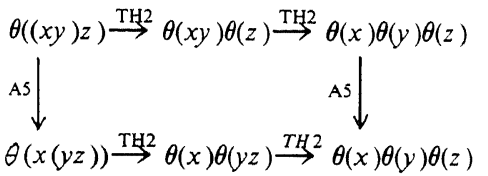


40. TH2 \& A7.

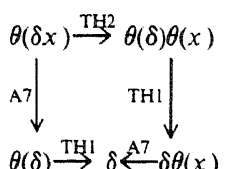

41. TH2 \& TH4.

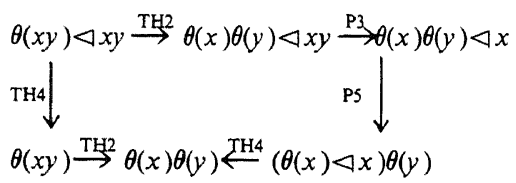

42. $\mathrm{TH} 3 \& \mathrm{Al}$.

$\theta(x+y) \stackrel{\text { IH } 3}{\rightarrow} \theta(x) \triangleleft y+\theta(y) \triangleleft x$

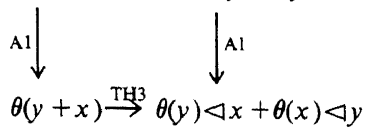

43. $\mathrm{TH} 3 \& \mathrm{~A} 2$.

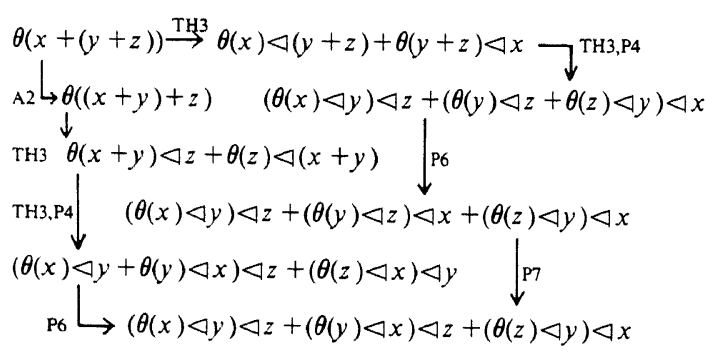

44. TH3 \& $\mathrm{A} 3$.

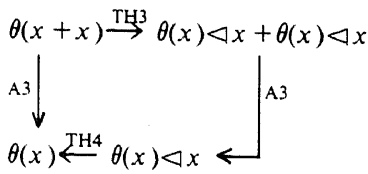

45. TH3 \& $\mathrm{A} 6$.

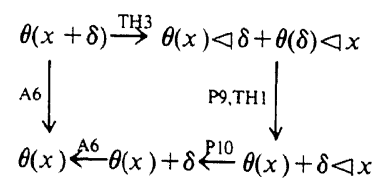

46. $\mathrm{TH} 3 \& \mathrm{TH} 4$ 


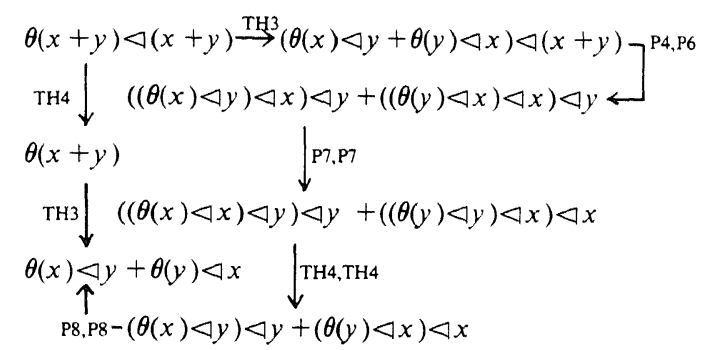

2.15 This completes the proof of 2.3.i, and thereby the proof of 2.3.

2.16 Theorem The following identities hold in the initial term model $A_{\omega}$ :

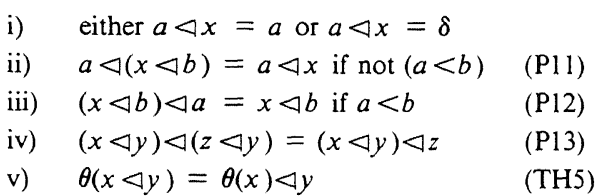

Proof All proofs are by induction on terms. An induction on $\mathrm{x}$ needs to consider only three cases: 1) $x \equiv a$, an atom; (2) $x \equiv a y$; (3) $x \equiv y+z$ (this uses 2.3.iii, we can eliminate $\varangle, \theta, \|, \mathbb{L}, \mid, \partial_{H}$ ).

i) Induction on $x$

case 1: suppose $x \equiv b$

case 1.1 not $(a<b)$. Then $a \triangleleft x=a \triangleleft b=a$

case $1.2 a<b$. Then $a \triangleleft x=a \triangleleft b=\delta$

case 2: $x \equiv y z$ and suppose (i) holds for $y$. We have $a \triangleleft x=a \triangleleft y z=a \triangleleft y$. Now apply the induction hypothesis.

case 3: $x \equiv y+z$ and suppose (i) holds for $y$ and $z$.

case 3.1 $a \triangleleft y=a$. Then $a \triangleleft x=a \triangleleft(y+z)=(a \triangleleft y) \triangleleft z=a \triangleleft z$. Now apply induction hypothesis. case $3.2 a \triangleleft y=\delta$. Then $a \triangleleft x=(a \triangleleft y) \triangleleft z=\delta \triangleleft z=\delta$.

ii) induction on $\mathrm{x}$. Suppose not $(a<b)$.

case 1: $x \equiv c$

case 1.1 not $(c<b)$. Then $a \triangleleft(c \triangleleft b)=a \triangleleft c$.

case $1.2 c<b$. Note that then we must have not $(a<c)$. So $a \triangleleft(c \triangleleft b)=a \triangleleft \delta=a=a \triangleleft c$.

case 2: $x \equiv c y . a \triangleleft(c y \triangleleft b)=a \triangleleft(c \triangleleft b) y=a \triangleleft(c \triangleleft b)=a \triangleleft c=a \triangleleft c y$.

case 3: $x \equiv y+z$, and suppose (ii) holds for $y$ and $z \cdot a \triangleleft((y+z) \triangleleft b)=a \triangleleft(y \triangleleft b+z \triangleleft b)=$ $(a \triangleleft(y \triangleleft b)) \triangleleft(z \triangleleft b)=(a \triangleleft y) \triangleleft(z \triangleleft b)=(a \triangleleft(z \triangleleft b)) \triangleleft y=(a \triangleleft z) \triangleleft y=a \triangleleft(y+z)$.

iii) induction on $\mathrm{x}$. Suppose $a<b$.

case 1: $x \equiv c$.

case 1.1 Not $(c<b)$. Note that then we must have not $(c<a)$. So $(c \triangleleft b) \triangleleft a=c \triangleleft a=c=c \triangleleft b$.

case $1.2 c<b .(c \triangleleft b) \triangleleft a=\delta \triangleleft a=\delta=c \triangleleft b$.

case 2: $x \equiv c y .(c y \triangleleft b) \triangleleft a=(c \triangleleft b) y \triangleleft a=((c \triangleleft b) \triangleleft a) y=(c \triangleleft b) y=c y \triangleleft b$

case 3: $x \equiv y+z$, and suppose (iii) holds for $y$ and $z .((y+z) \triangleleft b) \triangleleft a=(y \triangleleft b+z \triangleleft b) \triangleleft a=$ $(y \triangleleft b) \triangleleft a+(z \triangleleft b) \triangleleft a=y \triangleleft b+z \triangleleft b=(y+z) \triangleleft b$.

iv) Induction on $z$. 
case 1: $z \equiv a$.

case $1.1 a \triangleleft y=a$. Then $(x \triangleleft y) \triangleleft(a \triangleleft y)=(x \triangleleft y) \triangleleft a$.

case $1.2 a \triangleleft y=\delta$. In this case, we will prove the following variant of (iii) by induction on $y$ :

$\left(^{*}\right)$ if $a \triangleleft y=\delta$, then $(x \triangleleft y) \triangleleft a=x \triangleleft y$.

case 1.2.1 $y \equiv b$. This is (iii).

case 1.2.2 $y \equiv b w$. By 1.2 .1 we have $(x \triangleleft b w) \triangleleft a=(x \triangleleft b) \triangleleft a=x \triangleleft b=x \triangleleft b w$, for $\delta=a \triangleleft y=a \triangleleft b$.

case 1.2.3 $y \equiv w+v$. We have $\delta=a \triangleleft y=(a \triangleleft v) \triangleleft w$. We know by (i) that either $a \triangleleft v=a$ or $a \triangleleft v$ $=\delta$. If $a \triangleleft v=a$, we have $a \triangleleft w=\delta$. Thus we have either $a \triangleleft v=\delta$ or $a \triangleleft w=\delta$. Say $a \triangleleft v=\delta$ (the proof is very similar in the other case).

Then $(x \triangleleft y) \triangleleft a=(x \triangleleft(v+w)) \triangleleft a=((x \triangleleft v) \triangleleft w) \triangleleft a=((x \triangleleft v) \triangleleft a) \triangleleft w=(x \triangleleft v) \triangleleft w=$ $x \triangleleft(v+w)$.

Thus we have proved (*), and now $(x \triangleleft y) \triangleleft(a \triangleleft y)=(x \triangleleft y) \triangleleft \delta=x \triangleleft y=(x \triangleleft y) \triangleleft a$ follows. case 2: $z \equiv a w$. By case $1,(x \triangleleft y) \triangleleft(a w \triangleleft y)=(x \triangleleft y) \triangleleft(a \triangleleft y) w=(x \triangleleft y) \triangleleft(a \triangleleft y)=(x \triangleleft y) \triangleleft a=$ $(x \triangleleft y) \triangleleft a w$.

case 3: $z \equiv w+v$, and suppose (iv) holds for $w$ and $v$. Then $(x<y) \triangleleft((v+w) \triangleleft y)=$ $(x \triangleleft y) \triangleleft(v \triangleleft y+w \triangleleft y)=((x \triangleleft y) \triangleleft(v \triangleleft y)) \triangleleft(w \triangleleft y)=((x \triangleleft y) \triangleleft v) \triangleleft(w \triangleleft y)=((x \triangleleft y) \triangleleft(w \triangleleft y))$ $\triangleleft v=((x \triangleleft y) \triangleleft w) \triangleleft v=(x \triangleleft y) \triangleleft(w+v)$.

v) Induction on $x$.

case 1: $x \equiv a$.

case $1.1 a \triangleleft y=a$. Then $\theta(a \triangleleft y)=\theta(a)=a=a \triangleleft y=\theta(a) \triangleleft y$.

case $1.2 a \triangleleft y=\delta$. Then $\theta(a \triangleleft y)=\theta(\delta)=\delta=a \triangleleft y=\theta(a) \triangleleft y$

case 2: $x \equiv a z$. By case 1, $\theta(a z \triangleleft y)=\theta((a \triangleleft y) z)=\theta(a \triangleleft y) \theta(z)=(\theta(a) \triangleleft y) \theta(z)=\theta(a) \theta(z) \triangleleft y=$ $\theta(a z) \triangleleft y$.

case 3: $x \equiv z+w$ and suppose (v) holds for $z$ and $w$. Then $\theta((z+w) \triangleleft y)=\theta(z \triangleleft y+w \varangle y)=$ $\theta(z \triangleleft y) \triangleleft(w \triangleleft y)+\theta(w \triangleleft y) \triangleleft(z \triangleleft y)=(\theta(z) \triangleleft y) \triangleleft(w \triangleleft y)+(\theta(w) \triangleleft y) \triangleleft(z \triangleleft y)=$ (apply (iv)!) = $(\theta(z) \triangleleft y) \triangleleft w+(\theta(w) \triangleleft y) \triangleleft z=(\theta(z) \triangleleft w) \triangleleft y+(\theta(w) \triangleleft z) \triangleleft y=(\theta(z) \triangleleft w+\theta(w) \triangleleft z) \triangleleft y=$ $\theta(z+w) \triangleleft y$.

2.17 Note: Adding equations P1l-13 and TH5 to $\mathrm{RACP}_{\theta}$ as rewrite rules (reading from left to right) also gives a terminating and confluent rewrite system. The many and tedious details of the proof of this claim we happily leave to the reader.

\section{Simple examples}

We will now give some simple examples that use priorities, as defined in $\mathrm{ACP}_{\theta}$, and give some motivation of the choice of priorities.

We distinguish three cases:

1. give priority to interrupts;

2. give lower priority to time outs, error messages;

3. give priority to internal actions, real time behaviour.

3.1 Example 1:

Let $D$ be a finite set of data, and suppose we have an infinite sequence of data from $D$,

$$
\alpha=\left\langle d_{0}, d_{1}, d_{2}, \ldots\right\rangle
$$




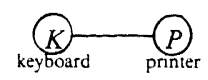

fig. 22

The printer will print these data, but can be interrupted by the keyboard. We have the following atomic actions $A$ : (a) actions of $\mathrm{K}$ :

1. $k(B R)=$ key in BREAK

2. $k(S P)=$ key in START PRINTING

3. $S(B R)=$ send $B R$ to the printer

4. $\quad s(S P)=$ send $S P$ to the printer

recursive equation for $\mathrm{K}$ :

$$
K=[k(B R) s(B R)+k(S P) s(S P)] K
$$

(b) actions of $\mathrm{P}$ :

1. $\quad p(d)=$ print symbol $d$;

2. $\quad r(B R)=$ receive $B R$ from the key-board;

3. $\quad r(S P)=$ receive $S P$ from the key-board.

The printer has two states, Printing and Waiting. We let $P_{i}$ stand for printing state after having printed $d_{0}, d_{1}, \ldots, d_{i-1}$; and $W_{i}$ stand for waiting state after having printed $d_{0}, d_{1}, \ldots, d_{i-1}(i \geqslant 0)$.

Equations for $\mathrm{P}$ :

$$
\begin{aligned}
& P=W_{0} \\
& W_{i}=r(S P) P_{i}+r(B R) W_{i} \\
& P_{i}=p\left(d_{i}\right) P_{i+1}+r(B R) W_{i}+r(S P) P_{i}
\end{aligned}
$$

3.2 Now we define the communication function by:

$$
\begin{aligned}
& s(B R) \mid r(B R)=b r \\
& s(S P) \mid r(S P)=s p
\end{aligned}
$$

and all other communications give $\delta$. We are interested in

$$
K \| P
$$

but want to hide unsuccessful communications. Therefore, we define

$$
H=\{s(B R), r(B R), s(S P), r(S P)\}
$$

and look at

$$
\partial_{H}(K \| P)
$$

Now we want to define a partial order an atomic actions giving priority. Note that both $b r$ and $s p$ should have priority over printing actions ( $b r$ must interrupt printing and $s(S P)$ must be received by the printer, otherwise the key-board will be blocked and a break cannot be given). All other priorities are given by 1.4.4. We have the following picture of the partial order (figure 23). 


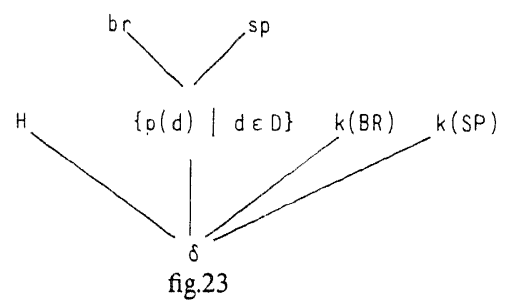

Here if two actions $a$ and $b$ are connected by a line, and $b$ is above $a$, then $a<b$. If two actions are not connected, they are incomparable. If we put a set at a certain position, it means each element of the set has that position. If $\theta$ is defined using this partial order, we can describe the system by:

$$
\theta \circ \partial_{H}(K \| P)
$$

3.3 Theorem. Put $\mathscr{P}_{i}=\theta \circ \partial_{H}\left(K \| P_{i}\right)$ and $\mho_{i}=\theta \circ \partial_{H}\left(K \| W_{i}\right)(i \geqslant 0)$, then we have the following equations:

1. $\varpi_{i}=k(B R) \cdot b r \cdot \mathscr{W}_{i}+k(S P) \cdot s p \cdot \mathscr{P}_{i}$.

2. $\mathscr{P}_{i}=p\left(d_{i}\right) \mathscr{P}_{i+1}+k(B R) \cdot b r \cdot \mathscr{W}_{i}+k(S P) \cdot s p \cdot \mathscr{P}_{i} .(i \geqslant 0)$.

Proof: as we go along, we will skip some steps. To calculate the merge, we need the expansion theorem (see Bergstra \& Tucker [5]) which goes as follows:

if $X_{1}, \ldots, X_{k}$ are given, put $\mathbf{X}^{i}=$ the merge of all $X_{n}$ except $X_{i}$ and $\mathbf{X}^{i, j}=$ the merge of all $X_{n}$ except $X_{i}$ and $X_{j}$. Then

$$
\text { (ET) } X_{i}\|\cdots\| X_{k}=\sum_{1 \leqslant i \leqslant k} X_{i} \llbracket \mathbf{X}^{i}+\sum_{1 \leqslant i<j \leqslant k}\left(X_{i} \mid X_{j}\right) \amalg \mathbf{X}^{i, j},
$$

i.e. we can start with an action of one of the processes, or with a communication between two of them.

Let $i \geqslant 0$.

$$
\begin{aligned}
\mathscr{W}_{i}=\theta \circ \partial_{H}\left(K \| W_{i}\right)= & \\
=\theta \circ \partial_{H}\left(k(B R)\left(s(B R) K \| W_{i}\right)\right. & + \\
+ & k(S P)\left(s(S P) K \| W_{i}\right)+ \\
& +r(B R)\left(K \| W_{i}\right)+ \\
& \left.+r(S P)\left(K \| P_{i}\right)\right)= \\
=\theta\left(k(B R) \cdot \partial_{H}\left(s(B R) K \| W_{i}\right)\right. & + \\
+ & \left.k(S P) \partial_{H}\left(s(S P) K \| W_{i}\right)\right)= \\
=k(B R) \cdot \theta \circ \partial_{H}\left(s(B R)\left(K \| W_{i}\right)\right. & +r(S P)\left(s(B R) K \| P_{i}\right)+ \\
& +r(B R)\left(s(B R) K \| W_{i}\right)+ \\
+ & \left.(s(B R) \mid r(B R))\left(K \| W_{i}\right)\right)+ \\
+k(S P) \cdot \theta \circ \partial_{H}\left(s(S P)\left(K \| W_{i}\right)\right. & +r(S P)\left(s(S P) K \| P_{i}\right)+ \\
+ & r(B R)\left(s(S P) K \| W_{i}\right)+ \\
+ & \left.(s(S P) \mid r(S P))\left(K \| P_{i}\right)\right)=
\end{aligned}
$$




$$
\begin{aligned}
& =k(B R) \theta\left(b r \cdot \partial_{H}\left(K \| W_{i}\right)\right)+k(S P) \theta\left(s p \cdot \partial_{H}\left(K \| P_{i}\right)\right)= \\
& =k(B R) \cdot b r \cdot W_{i}+k(S P) \cdot s p \cdot \mathscr{P}_{i} \text {. } \\
& \mathscr{\rho}_{i}=\theta \circ \partial_{H}\left(K \| P_{i}\right)= \\
& =\theta\left(k(B R) \cdot \partial_{H}\left(s(B R) K \| P_{i}\right)+\right. \\
& +k(S P) \cdot \partial_{H}\left(s(S P) K \| P_{i}\right)+ \\
& \left.+p\left(d_{i}\right) \partial_{H}\left(K \| P_{i+1}\right)\right)= \\
& =k(B R) \cdot \theta\left(p\left(d_{i}\right) \partial_{H}\left(s(B R) K \| P_{i+1}\right)+\right. \\
& \left.+(s(B R) \mid r(B R)) \cdot \partial_{H}\left(K \| W_{i}\right)\right)+ \\
& +k(S P) \cdot \theta\left(p\left(d_{i}\right) \cdot \partial_{H}\left(s(S P) K \| P_{i+1}\right)+\right. \\
& \left.+(s(S P) \mid r(S P)) \cdot \partial_{H}\left(K \| P_{i}\right)\right)+ \\
& +p\left(d_{i}\right) \mathscr{P}_{i+1}= \\
& =\text { (this is where we use the priority) } \\
& k(B R) \cdot b r \cdot \mathscr{W S}_{i}+k(S P) \cdot s p \cdot \mathscr{P}_{i}+p\left(d_{i}\right) \cdot \mathscr{P}_{i+1} \text {. }
\end{aligned}
$$

We can make the following state transition diagram:

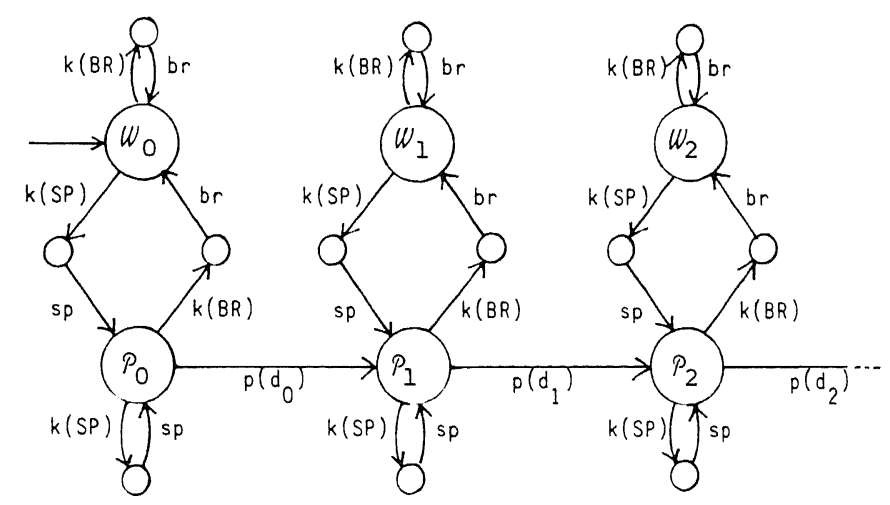

fig. 24

3.4 Example 2: Suppose we have a file $F$ containing an infinite sequence of data from a finite set $D$, so $F$ contains

$$
\alpha=\left\langle d_{0}, d_{1}, \ldots\right\rangle \text {. }
$$

These data can be sent to the printer and subsequently printed, or a file crash might occur, in which case an error statement will be generated.

$$
\begin{gathered}
\text { file } \\
\text { fig. } 25
\end{gathered}
$$


We have the following atomic actions $A$ :

(a) actions of $F$ :

1. $g(d)=$ get the next symbol, $d \in D$, from the file

2. $s(d)=$ send $d \in D$ to the printer

3. $c r=$ file crash.

Let $F_{i}$ stand for the state of $F$ after $d_{0}, \ldots, d_{i-1}$ have been sent $(i \geqslant 0)$. Then $F$ is described by the following equations:

$$
\begin{aligned}
& F=F_{0} \\
& F_{i}=g\left(d_{i}\right) s\left(d_{i}\right) \cdot F_{i+1}+c r \quad(i \geqslant 0) .
\end{aligned}
$$

(b) actions of $P$ :

1. $\quad r(d)=$ receive $d \in D$ from the file

2. $p(d)=$ print $d \in D$

3. $\quad o(C R)=$ observe file crash

4. $\quad p(C R)=$ print 'FILE HAS CRASHED'.

Recursive equation for $P$ :

$$
P=\sum_{d \in D} r(d) \cdot p(d) \cdot P+o(C R) \cdot p(C R)
$$

(c) communication actions:

if $d \in D$, then $r(d) \mid s(d)=c(d)$ (communicate $d$ ). All other communications give $\delta$. Since we want to hide all unsuccessful communications, we define $H=\{r(d), s(d) \mid d \in D\}$, and look at

$$
\partial_{H}(F \| P) \text {. }
$$

3.5 Priority. Here the rationale of defining priorities is different. The file-crash $c r$ might occur at any moment, but $o(C R)$, the observation of a file-crash, can only occur if the file has actually crashed. We ensure this by giving $o(C R)$ a lower priority than every 'regular' action of $F$, so we must have $o(C R)<g(d)$ and $o(C R)<c(d)$. This gives the following picture (figure 26).

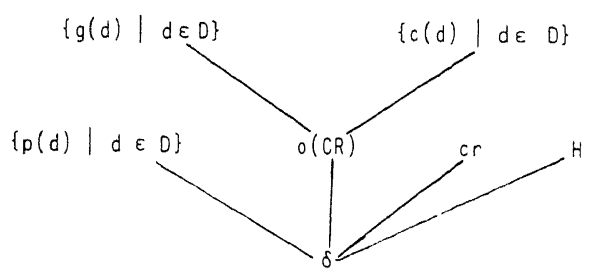

fig. 26

If $\theta$ is defined using this partial order, we can describe the system by:

$$
\theta \circ \partial_{H}(F \| P)
$$

3.6 Theorem: Put $\mathscr{F}_{i}=\theta \circ \partial_{H}\left(F_{i} \| P\right)$, and $\mathscr{P}_{i}=\theta \circ \partial_{H}\left(F_{i+1} \| p\left(d_{i}\right) P\right)(i \geqslant 0)$, then we have the following equations: 
1) $\quad \mathscr{F}_{i}=g\left(d_{i}\right) \cdot c\left(d_{i}\right) \cdot \mathscr{P}_{i}+c r . o(C R) \cdot p(C R)$

2) $\quad \mathscr{P}_{i}=g\left(d_{i+1}\right) \cdot p\left(d_{i}\right) \cdot c\left(d_{i+1}\right) P_{i+1}+$

$+\operatorname{cr} \cdot p\left(d_{i}\right) \cdot o(C R)_{\cdot p}(C R)+p\left(d_{i}\right) \mathscr{Y}_{i+1}(i \geqslant 0)$

Proof let $i \geqslant 0$.

$$
\begin{aligned}
& \mathscr{F}_{i}=\theta \circ \partial_{H}\left(F_{i} \| P\right) \\
& =\theta\left(g\left(d_{i}\right) \cdot \partial_{H}\left(s\left(d_{i}\right) F_{i+1} \| P\right)+c r \cdot \partial_{H}(P)+\right. \\
& \left.+o(C R) \cdot \partial_{H}\left(F_{i} \| p(C R)\right)\right)= \\
& =\left(\text { use } g\left(d_{i}\right)>o(C R)\right) \\
& g\left(d_{i}\right) \cdot \theta\left(\left(s\left(d_{i}\right) \mid r\left(d_{i}\right)\right) \partial_{H}\left(F_{i+1} \| p\left(d_{i}\right) P\right)\right. \\
& \left.+o(C R) \cdot \partial_{H}\left(s\left(d_{i}\right) F_{i+1} \| p(C R)\right)\right)+ \\
& +c r \cdot \theta(o(C R) \cdot p(C R))= \\
& =\left(\text { use } c\left(d_{i}\right)>o(C R)\right) \\
& g\left(d_{i}\right)_{\circ} c\left(d_{i}\right) \cdot \mathscr{P}_{i}+c r . o(C R) \cdot p(C R) . \\
& \mathscr{P}_{i}=\theta \circ \partial_{H}\left(F_{i+1} \| p\left(d_{i}\right) P\right)= \\
& =\theta\left(g\left(d_{i+1}\right) \cdot \partial_{H}\left(s\left(d_{i+1}\right) F_{i+2} \| p\left(d_{i}\right) P\right)+\right. \\
& +c r . \partial_{H}\left(p\left(d_{i}\right) P\right)+ \\
& \left.+p\left(d_{i}\right) \cdot \partial_{H}\left(F_{i+1} \| P\right)\right)= \\
& =g\left(d_{i+1}\right) \cdot \theta\left(p\left(d_{i}\right) \cdot \partial_{H}\left(s\left(d_{i+1}\right) F_{i+2} \| P\right)\right)+ \\
& +c r \cdot p\left(d_{i}\right) \cdot o(C R) \cdot p(C R)+ \\
& +p\left(d_{i}\right) \cdot \mathscr{F}_{i+1}= \\
& =g\left(d_{i+1}\right) \cdot p\left(d_{i}\right) \cdot \theta\left(c\left(d_{i+1}\right) \cdot \partial_{H}\left(F_{i+2} \| p\left(d_{i+1}\right) P\right)+\right. \\
& +o(C R) \cdot \partial_{H}\left(s\left(d_{i+1} F_{i+2} \| p(C R)\right)\right)+ \\
& +c r \cdot p\left(d_{i}\right) \cdot o(C R) \cdot p(C R)+p\left(d_{i}\right) \cdot \sigma_{i} F_{i+1}= \\
& =\left(\text { use } c\left(d_{i+1}\right)>o(C R)\right) \\
& g\left(d_{i+1}\right) \cdot p\left(d_{i}\right) \cdot c\left(d_{i+1}\right) \mathscr{P}_{i+1}+ \\
& +\operatorname{cr} \cdot p\left(d_{i}\right) \cdot o(C R) \cdot p(C R)+p\left(d_{i}\right) \cdot \cdot \cdot r_{i}+1 .
\end{aligned}
$$

State transition diagram: 


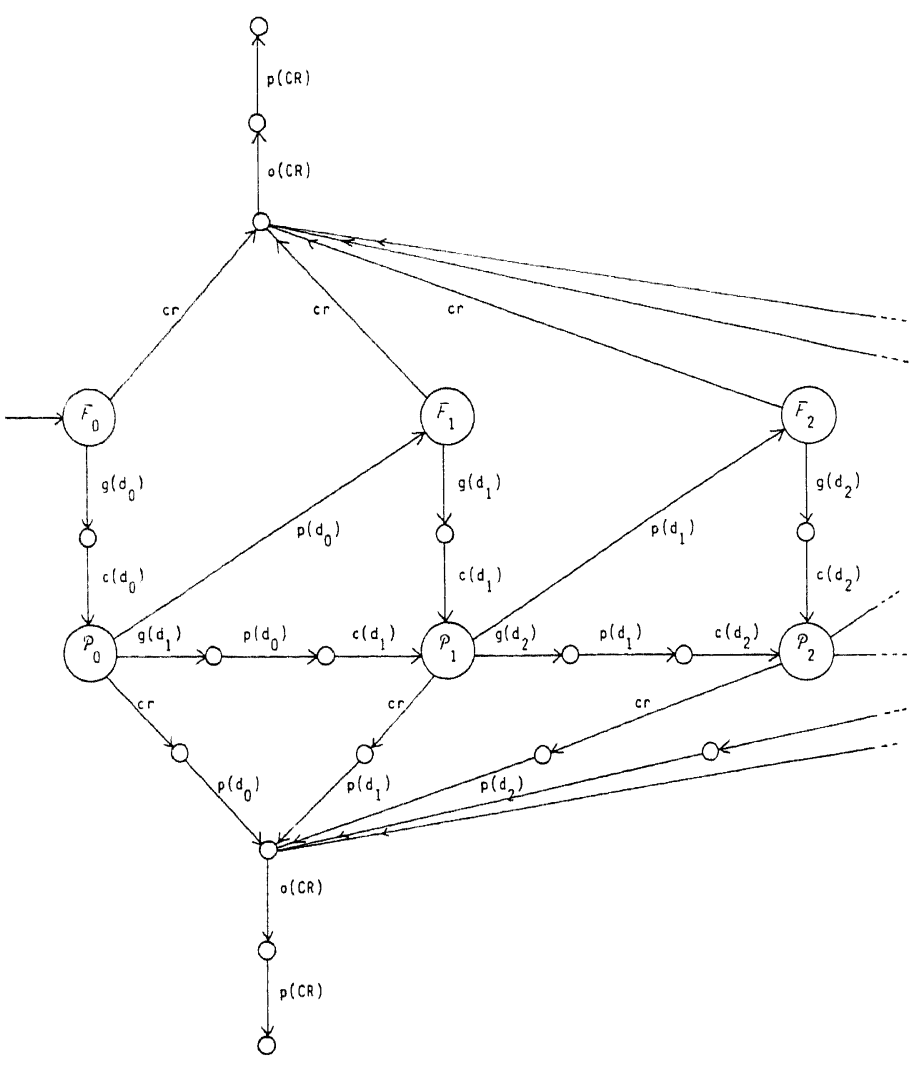

fig. 27

3.7 Now we want to focus on the printing actions, in the system just described, and abstract from the other actions. This will give an easy equation for the system. The tool to carry out abstractions is $\mathrm{ACP}_{r}$, which is ACP with an abstraction operator $\tau_{I}$ and silent steps $\tau$ (see (Bergstra \& Klop [2]).

Since we do not want to mix $\mathrm{ACP}_{\theta}$ and $\mathrm{ACP}_{\tau}$, we will assume that all $\theta$ and $\triangleleft$ are eliminated from terms like $\mathscr{F}_{i}$ and $\mathscr{P}_{i}$ (possible by 2.3.iii), so that they become ACP-terms. The axiom system $\mathrm{ACP}_{\tau}$ is presented on the next page. (table 4)

3.8 Abstraction Define $I=\{c(d), g(d) \mid d \in D\} \cup\{c r, o(C R)\}$ and look at

$$
\tau_{I}^{\circ \theta \circ \partial_{H}(F \| P)}
$$

From theorem 3.6, we obtain the following equations:

1) $\tau_{I}\left(\mathscr{G}_{i}\right)=\tau\left(\tau \tau_{I}\left(\mathscr{P}_{i}\right)+\tau p(C R)\right)$

2) $\tau_{I}\left(\mathscr{P}_{i}\right)=\tau\left(\tau p\left(d_{i}\right) \cdot \tau_{I}\left(\mathscr{P}_{i+1}\right)+\tau p\left(d_{i}\right) \cdot p(C R)+p\left(d_{i}\right) \tau_{I}\left(\mathscr{F}_{i+1}\right)\right)$. 
$A C P_{\tau}$

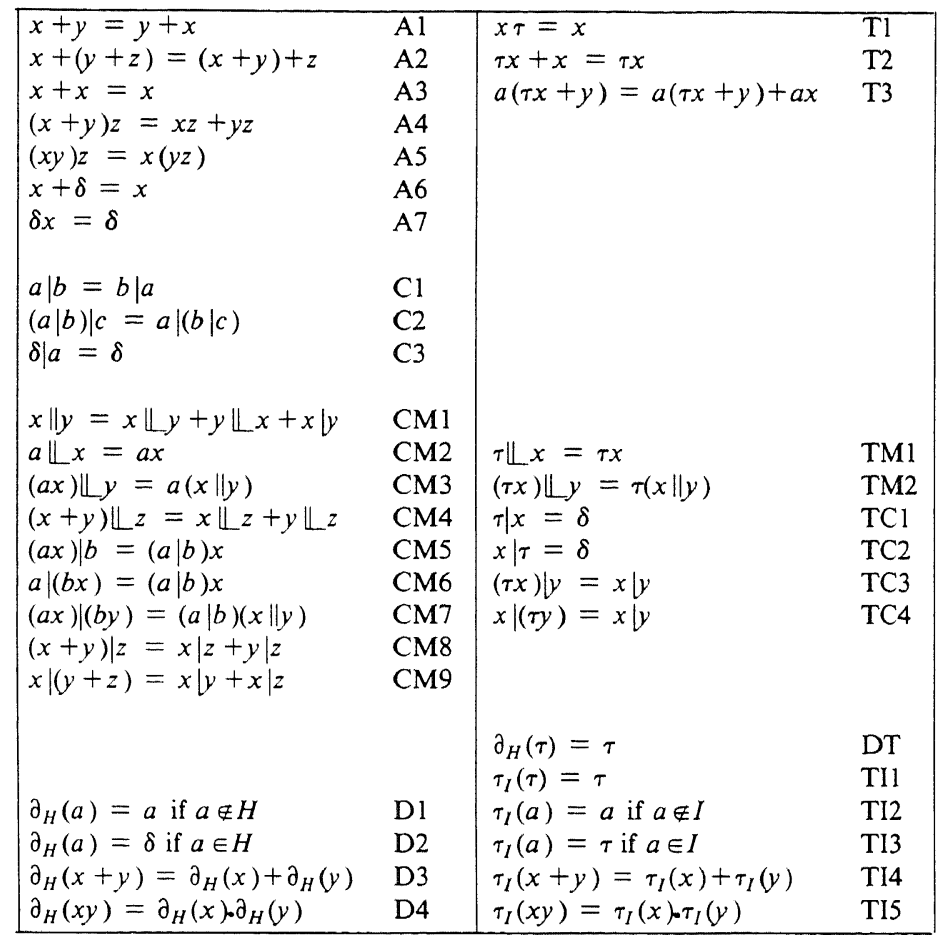

3.9 Example 3 Let us now modify the previous example by changing the priority ordering in the following way:

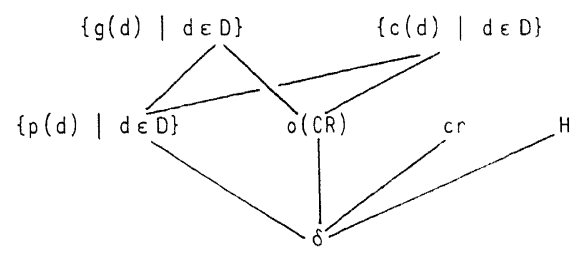

$$
\text { fig. } 28
$$

This expresses that in real time the 'internal' actions $g(d)$ and $c(d)$ will always precede the external action $p(d)$. This is a simplifying assumption about the real time behaviour of the system and its environment.

Now theorem 3.6 turns into:

3.10 Theorem (abbreviations as in 3.6):

1) $\mathscr{F}_{i}=g\left(d_{i}\right) \cdot c\left(d_{i}\right) \mathscr{P}_{i}+c r . o(C R) \cdot p(C R)$

2) $\mathscr{P}_{i}=g\left(d_{i+1}\right) \cdot p\left(d_{i}\right) \cdot c\left(d_{i+1}\right) \mathscr{P}_{i+1}+c r \cdot p\left(d_{i}\right) \cdot o(C R) \cdot p(C R) . \quad(i \geqslant 0)$.

Now $\mathscr{F}_{i}$ has disappeared from the second equation, so this simplifies to:

$\left.1^{\prime}\right): \mathscr{\mathscr { F }}_{0}=g\left(d_{0}\right) \cdot c\left(d_{0}\right) \cdot \mathscr{P}_{0}+c r . o(C R) \cdot p(C R)$

2 as above, and the following state transition diagram (compare with the complicated diagram in 3.6) 


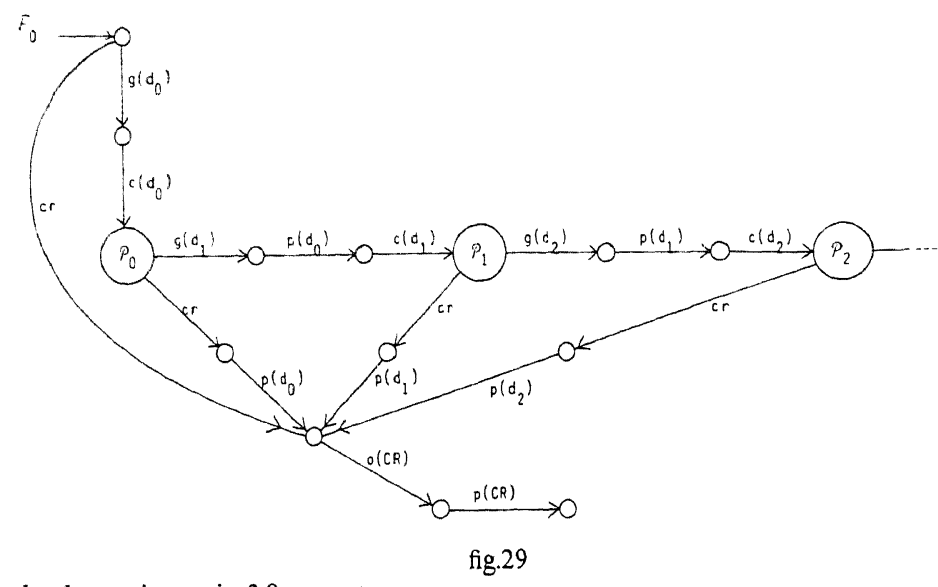

When we do abstraction as in 3.8 , we get

$\left.1^{\prime}\right): \quad \tau_{I}\left(\mathscr{T}_{0}\right)=\tau\left(\tau_{\cdot} \tau_{I}\left(\oplus_{0}\right)+\tau \cdot p(C R)\right)$

2) $\tau_{I}\left(\mathscr{P}_{i}\right)=\tau\left(\tau \cdot p\left(d_{i}\right) \cdot \tau_{I}\left(\oplus_{i+1}\right)+\tau \cdot p\left(d_{i}\right) \cdot p(C R)\right)$

\section{Example: a toy distributed system}

\subsection{Set-up}

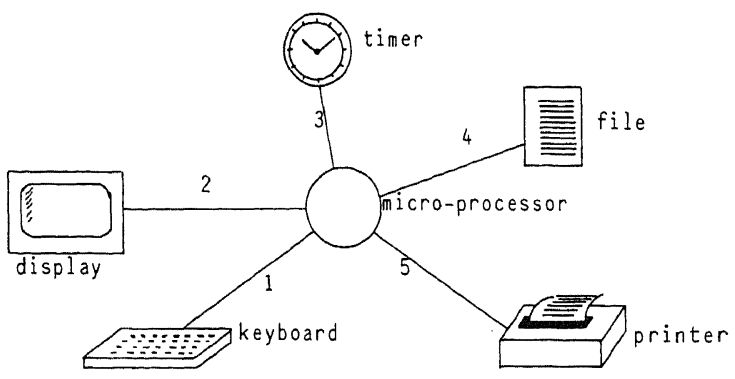

fig. 30

Description: At a command from the key-board, a word (string of data) will be released from a file holding infinitely many words, and sent to the printer. Then the word can be printed symbol by symbol. At a signal from the timer, the status of the printer will be requested and the answer displayed. Printing can be interrupted by a BREAK from the key-board.

4.2 Aim We want to give a description of this system, using process algebra. First we will give recursive equations defining each component of the system. Then we will look at the free merge of these processes, will encapsulate unsuccesful communications and give priority to interrupts. Next, we will give recursive equations for the whole system, and then, in order to focus on certain aspects of the system, we will abstract from other elements. We present three ways of doing that.

4.3 Description of components, using state transition diagrams (or process graphs).

4.3.1 Keyboard $K$. The key-board can generate a message, and send it along channel 1 . After that, it is back in its original state. 


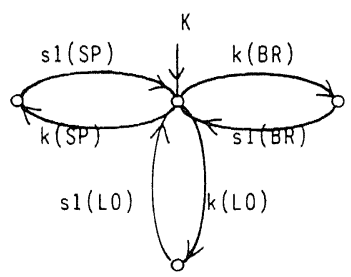

fig.31

actions of $K: 1 \cdot k(B R)=$ key in BREAK (will stop printing); 2. $k(L O)=$ key in LOAD (will get a word from the file and load it into the memory of the printer); 3. $k(S P)=$ key in START PRINTING (will cause the printer to start); 4, 5, 6: $s 1(B R), s 1(L O), s 1(S P)=$ send $B R, L O, S P$ along channel 1.

Recursive equation for $K$ :

$K=(k(B R) \cdot s 1(B R)+k(L O) \cdot s 1(L O)+k(S P) \cdot s 1(S P)) K$.

4.3.2 Display $D$. The display can receive a message along channel 2 and display it. After that, it is back in its original state.

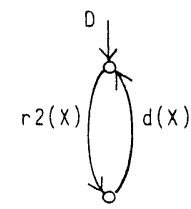

$$
\begin{aligned}
X= & P E, P W, P P, P L, P D, P R, F E, N D, N R \\
& \text { (the diagram actually consists } \\
& \text { of } 9 \text { loops as shown) }
\end{aligned}
$$

fig. 32

actions of $D: 1 . d(P E)=$ display: PRINTER ERROR

2. $d(P W)=$ display: PRINTER WAITING

3. $d(P P)=$ display: PRINTER PRINTING

4. $d(P R)=$ display: PRINTER READY

5. $d(P L)=$ display: PRINTER LOADED, PLEASE KEY IN $S P$.

6. $d(P D)=$ display: PRINTER DONE, PLEASE KEY IN LOAD

7. $d(F E)=$ display: FILE ERROR

8. $d(N D)=$ display: PRINTER NOT DONE, PLEASE WAIT

9. $d(N R)=$ display: PRINTER NOT READY, PLEASE WAIT

10-18. $r 2(X)=$ receive message $X$ along channel $2(X=P E, P W, P P, P R, P L, P D, F E, N D, N R)$ Here $P W, P P, P R(2-4)$ are status reports from the printer; $P L, P D(5,6)$ are messages from the printer that it has completed a certain phase; $P E, F E(1,7)$ are error messages, generated when file or printer do not respond; $N D, N R(8,9)$, are messages, generated, when $L O$ or $S P$ is keyed in too early.

Recursive equation for $D$ :

$$
D=\left(\sum_{\substack{X=P E, P W . P P, P R \\ P L, P D, F E, N D, N R}} r 2(X) \cdot d(X)\right) D
$$

4.3.3 Timer $T$. The timer can tick, and then it sends a message along channel 3. After that, it is back in its original state. 


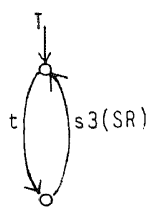

fig.33

actions of $T: 1 . t=$ TICK;

2. $s 3(S R)=$ send message STATUS REPORT? along channel 3 (will ask for a status report of the printer).

Recursive equation for $T$ :

$$
T=\operatorname{tos} 3(S R) \cdot T
$$

4.3.4 File $F$. The file holds infinitely many words of length $N$. Say $\mathbb{D}$ is a finite set of data (maybe containing a blank), then we let $\mathbb{D}^{N}$ stand for the set of all words of length $N$. At the request LOAD, $F$ will release the next word and send it to the printer.

If $F=\left\langle x_{1}, x_{2}, \ldots\right\rangle$ (the original state), we put $F_{i}=\left\langle x_{i+1}, x_{i}+2, \ldots\right\rangle(i \in \mathbb{N}$, the state after releasing $i$ words (the $x_{i}$ are in $\mathbb{D}^{N}$ ).

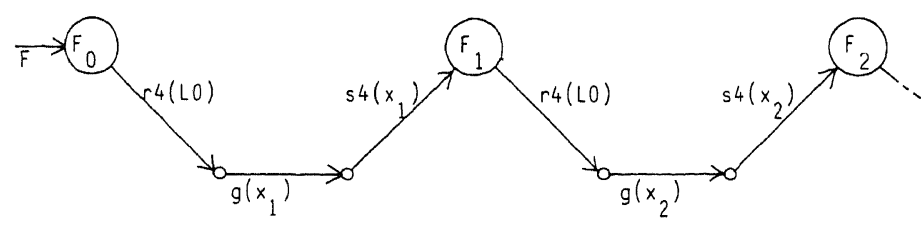

fig.34

In this diagram, we use the following convention: if, inside a node we put a symbol $S$, then by $S$ we mean the subgraph with $S$ as root.

actions of $\mathrm{F}$ :

1. $r 4(L O)=$ receive message LOAD along channel 4 ;

2. $g(x)=$ get the next word $x \in \mathbb{D}^{N}$ from the file

3. $s 4(x)=$ send $x \in \mathbb{D}^{N}$ along channel 4 .

Equations for $F$ :

$$
\begin{gathered}
F=F_{0} \\
F_{i}=r 4(L O) \cdot g\left(x_{i+1}\right) \cdot s 4\left(x_{i+1}\right) F_{i+1} \quad(i \in \mathbb{N}) .
\end{gathered}
$$

4.3.5 Printer $P$

The printer has three basic states:

1. waiting, with printing queue empty $\left(P_{\epsilon}\right)$;

2. ready, with printing queue a word $x \in \mathbb{D}^{N}$ (printing has not started yet) $\left(R_{x}\right)$;

3. printing, with printing queue a word $x \in \mathbb{D}^{\leqslant N}=\underset{n=1, \ldots, N}{\cup} \mathbb{D}^{n}\left(P_{x}\right)$.

The following is a state transition diagram, using a word $x=d_{1} \ldots . d_{N} \in \mathbb{D}^{N}$. 
actions of $P$ :

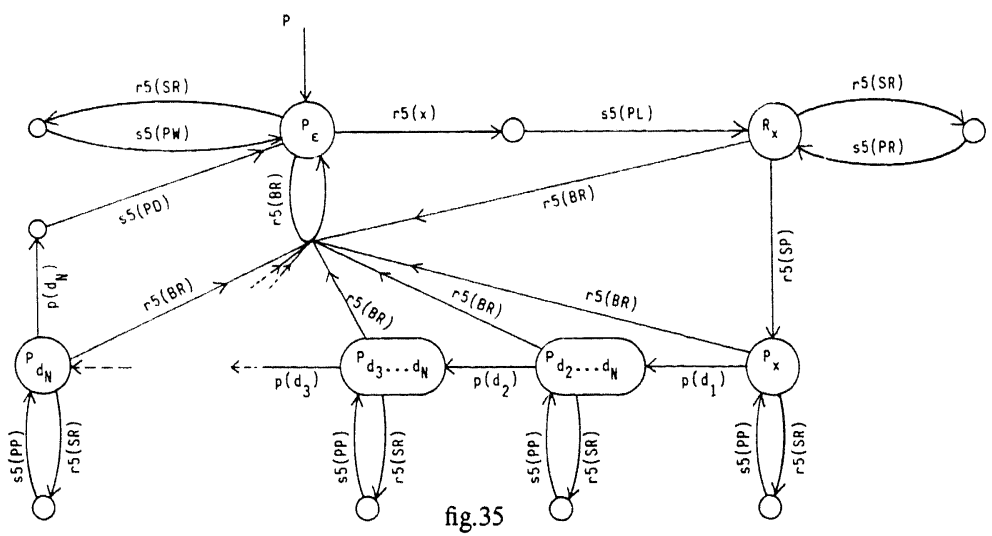

1. $r 5(x)=$ receive $x \in \mathbb{D}^{N}$ along channel 5;

2. $r 5(B R)=$ receive BREAK along 5 ;

3. $r 5(S R)=$ receive STATUS REPORT? along 5 ;

4. $s 5(P W)=$ send PRINTER WAITING along 5; ] (answers

5. $s 5(P R)=$ send PRINTER READY along $5 ; \quad\}$ to $S R$ )

6. $s 5(P P)=$ send PRINTER PRINTING along 5 ;

$\left.\begin{array}{l}\text { 7. } s 5(P L)=\text { send PRINTER LOADED along } 5 ; \\ \text { 8. } s 5(P D)=\text { send PRINTER DONE along } 5 ;\end{array}\right\}$ (state changes)

9. $p(d)=$ print $d \in \mathbb{D}$.

Recursive equations for $P$ :

i) $P=P_{\mathrm{c}}=\Sigma_{x \in \mathbf{D}^{N}} r 5(x) s 5(P L) R_{x}+(r 5(S R) s 5(P W)+r 5(B R)) P_{c}$

ii) for all $x \in \mathbb{D}^{N}$

$$
R_{x}=r 5(S R) s 5(P R) R_{x}+r 5(S P) P_{x}+r 5(B R) P_{\epsilon}
$$

iii)for all $x \in \mathbb{D}^{n}, n=2, \ldots, N, x=d_{1} \cdots d_{n}$ :

$$
P_{x}=r 5(S R) s 5(P P) P_{x}+p\left(d_{1}\right) P_{d_{2} \cdots d_{n}}+r 5(B R) P_{\epsilon}
$$

iv)for all $d \in \mathbb{D}$ :

$$
P_{d}=r 5(S R) s 5(P P) P_{d}+(p(d) s 5(P D)+r 5(B R)) P_{c} .
$$

4.3.6 Micro-processor $M$ :

In $M$ we need three states, according to the state of the printer, so:

$M_{w}=$ state when printer is waiting;

$M_{r}=$ state when printer is ready;

$M_{p}=$ state when printer is printing.

actions of $M$ are all communication actions; description of letter codes are given elsewhere.

1,2,3:rl(BR), $r 1(L O), r 1(S P)$;

4-12:s 2(PE), s2(PW), s2(PP), s2(PR), s2(PL), s2(PD), s2(FE), s2(ND), s2(NR);

13:r3(SR);

$14: s 4(L O)$;

$15: r 4(x)\left(x \in \mathbb{D}^{N}\right)$;

16-18:s $5(x)\left(x \in \mathbb{D}^{N}\right) ; s 5(B R), s 5(S R)$;

19-23:r5(PW), r5(PR), r5(PP), r5(PL), r5(PD) 


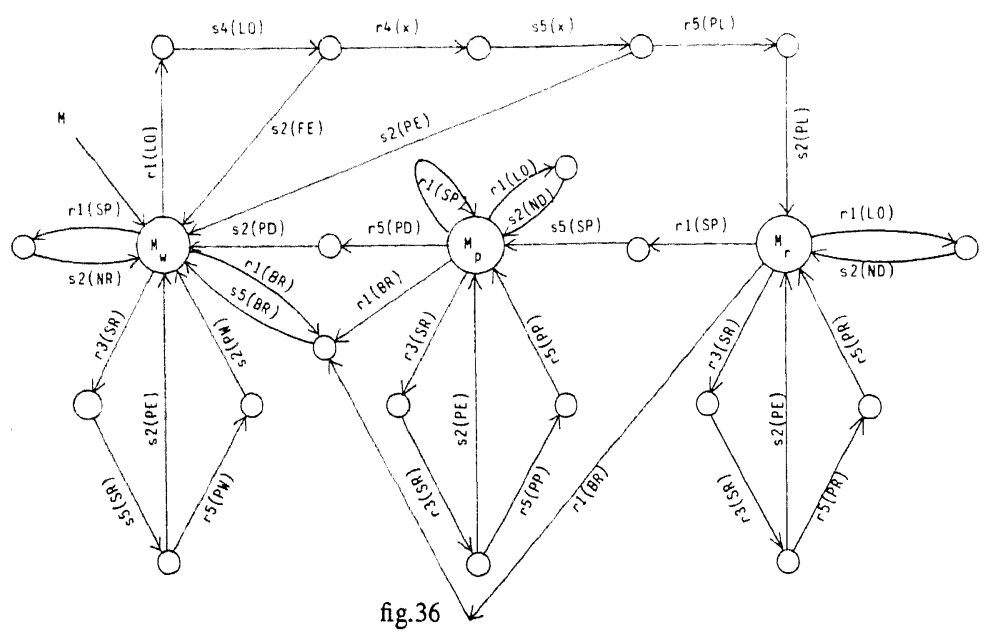

Recursive equations for $M$ :

$$
\begin{aligned}
& \text { i) } M=M_{w}=[r 3(S R) s 5(S R)(s 2(P E)+r 5(P W) s 2(P W))+ \\
& +r 1(S P) s 2(N R)+r 1(B R) s 5(B R)] M_{w}+ \\
& +r 1(L O) s 4(L O)\left[s 2(F E) M_{w}+\right. \\
& \left.+\sum_{x \in \mathbf{0}^{N}} r 4(x) s 5(x)\left(s 2(P E) M_{w}+r 5(P L) s 2(P L) M_{r}\right)\right] \\
& \text { ii }) M_{r}=\left(r 1(L O) s 2(N D)+r 3(S R) s 5(S R)(s 2(P E)+r 5(P R) s 2(P R)) M_{r}+\right. \\
& +r 1(S P) s 5(S P) M_{p}+r 1(B R) s 5(B R) M_{w} \\
& \text { iii }) M_{p}=(r 1(S P)+r l(L O) s 2(N D)+r 3(S R) s 5(S R)(s 2(P E)+r 5(P P) s 2(P P))) M_{p} \\
& +(r 1(B R) s 5(B R)+r 5(P D) s 2(P D)) M_{w} .
\end{aligned}
$$

4.4 We have now described each component, and now we want to study the interleaving of these processes, i.e.$$
K\|D\| T\|F\| P \| M
$$

When we expand this term, we have to know the communication function on actions. Basically, only $\operatorname{ri}(X) \mid \operatorname{si}(X)\left(i=1,2,3,4,5\right.$ and $X$ a two-letter code or a word in $\left.\mathbb{D}^{N}\right)$ give non- $\delta$ communications. Specifically:

i) $r \mathrm{l}(B R)|s \mathrm{l}(B R)=r 5(B R)| s 5(B R)=b r$ (break)

ii) $r 3(S R)|s 3(S R)=r 5(S R)| s 5(S R)=$

$$
\begin{aligned}
& =r 5(P W)|s 5(P W)=r 2(P W)| s 2(P W)= \\
& =r 5(P R)|s 5(P R)=r 2(P R)| s 2(P R)= \\
& =r 5(P P)|s 5(P P)=r 2(P P)| s 2(P P)=s r \text { (status report) }
\end{aligned}
$$

iii) $r 1(L O)|s 1(L O)=r 4(L O)| s 4(L O)=10 \quad(\mathrm{load})$

iv) $r \mathrm{l}(S P)|s \mathrm{l}(S P)=r 5(S P)| s 5(S P)=s p$ (start printing)

v) $r 5(P D)|s 5(P D)=r 2(P D)| s 2(P D)=r 5(P L) \mid s 5(P L)=$

$$
=r 2(P L)|s 2(P L)=r 2(N D)| s 2(N D)=r 2(N R) \mid s 2(N R)
$$

$$
=p m \text { (printer message) }
$$


vi) $r 2(P E)|s 2(P E)=r 2(F E)| s 2(F E)=e m$ (error message)

vii)for $x \in \mathbb{D}^{N}$

$$
r 4(x)|s 4(x)=r 5(x)| s 5(x)=s d(x) \text { (send data) }
$$

viii)all other communications (i.e. those not defined by i-vii above or rule $C 1$ ) are $\delta$.

4.5 Then, we want to throw away (encapsulate) all unsuccessful communications, so if we define $H$ to be the set of all $\operatorname{ri}(X)$ and all $\operatorname{si}(X)\left(i=1,2,3,4,5 ; X\right.$ a two-letter code or a word in $\left.\mathbb{D}^{N}\right)$, we want to look at

$$
\partial_{H}(K\|D\| T\|F\| P \| M)
$$

4.6 Priorities We will define priorities in a certain way, so that strings of actions that belong together, will be executed together, so that the formulation of theorem 4.8 becomes readable. The principles behind this formulation are explained in section 3 .

Define $E=\{t, k(B R), k(L O), k(S P)\} \cup\{p(d) \mid d \in D\}$ ('external' actions) and

$$
\begin{gathered}
C=\{p m, b r, l o, s p, s r\} \cup\left\{s d(x), g(x) \mid x \in \mathbb{D}^{N}\right\} \\
\cup\{d(X) \mid X=P E, P W, P P, P R, P L, P D, F E, N D, N R\}
\end{gathered}
$$

(communication actions and other 'internal' actions), then we have the following picture:

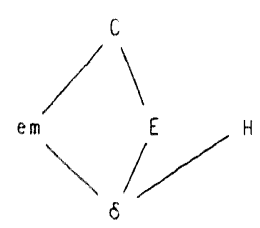

(conventions as explained in 3.2).

$$
\text { fig. } 37
$$

If $\theta$ is defined with respect to this partial ordering, we look at

$$
\text { थर्ण }=\theta^{\circ} \partial_{H}(K\|D\| T\|F\| P \| M)
$$

4.7 Now we will prove some recursive equations that hold for the whole system $" \mathrm{Ul}_{0}$. First some abbreviations:

$$
\mathscr{W l}_{i}=\theta^{\circ} \partial_{H}\left(K\|D\| T\left\|F_{1}\right\| P_{e} \| M_{w}\right) \quad(i \in \mathbb{N})
$$

(printer is waiting and $i$ words have been handled)

$$
\operatorname{ch}_{\imath}=\theta \circ \partial_{H}\left(K\|D\| T\left\|F_{i}\right\| R_{x_{1}} \| M_{r}\right) \quad(i \geqslant 1)
$$

(printer is loaded, the $i$ - th word is being handled)

$$
\operatorname{cop}_{1}^{n}=\theta \cdot \dot{\partial}_{H}\left(K\|D\| T\left\|F_{1}\right\| P_{x} \| M_{p}\right) \quad(i \geqslant 1, n=1, \ldots, N,
$$

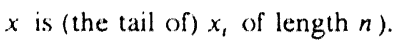

(printer is printing the $i-$ th word, still $n$ characters to go)

$$
\iota_{1}^{0}=\theta^{\circ} \partial_{H}\left(K\|D\| T\left\|F_{1}\right\|(s 5(P D) P) \| M_{p}\right) \quad(i \geqslant 1)
$$

(printer has just printed the last character of the $i$-th word)

\subsection{Theorem:}

1. จा

$+k(L O)$ lo.lo.g( $\left.x_{1}+1\right) \cdot \operatorname{sd}\left(x_{1}+1\right) \cdot s d\left(x_{1}+1\right) \cdot p m \cdot p m \cdot d(P L) \cdot R_{1+1}+$

$+k(S P)$ sp.pm.d $(N R) \cdot$ oul $_{t}+$

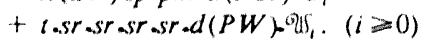




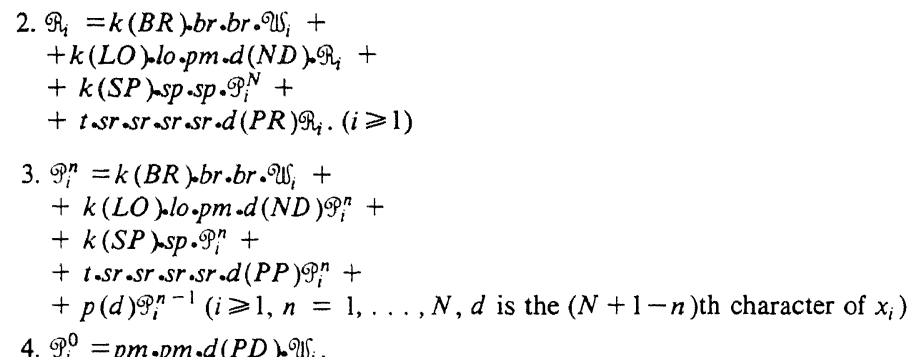

Proof: We use ET as in the proof of 3.3 .

1. Let $i \geqslant 0$

$$
\begin{aligned}
& \mathscr{w}_{i}=\theta_{\circ} \circ \partial_{H}\left(K\|D\| T\left\|F_{i}\right\| P_{\epsilon} \| M_{w}\right)= \\
& \theta \circ \partial_{H}\left(k(B R) \cdot\left((s 1(B R) K)\|D\| T\left\|F_{i}\right\| P_{c} \| M_{w}\right)+\right. \\
& +k(L O) \cdot\left((s 1(L O) K)\|D\| T\left\|F_{i}\right\| P_{c} \| M_{w}\right)+ \\
& +k(S P) \cdot\left((s 1(S P) K)\|D\| T\left\|F_{i}\right\| P_{\epsilon} \| M_{w}\right)+ \\
& +r 2(P E)_{-}\left(K\|(d(P E) D)\| T\left\|F_{i}\right\| P_{c} \| M_{w}\right)+ \\
& +r 2(P W) \cdot\left(K\|(d(P W) D)\| T\left\|F_{i}\right\| P_{\epsilon} \| M_{w}\right)+ \\
& +r 2(P P)_{-}\left(K\|(d(P P) D)\| T\left\|F_{i}\right\| P_{\epsilon} \| M_{w}\right)+ \\
& \text { - - - }(\text { six more } r 2(X))-\cdots-- \\
& +r 2(N R)_{\cdot}\left(K\|(d(N R) D)\| T\left\|F_{i}\right\| P_{c} \| M_{w}\right)+ \\
& +t \cdot\left(K\|D\|(s 3(S R) T)\left\|F_{i}\right\| P_{\epsilon} \| M_{w}\right)+ \\
& +r 4(L O) \cdot\left(K\|D\| T\left\|\left(g\left(x_{i+1}\right) s 4\left(x_{i+1}\right) F_{i+1}\right)\right\| P_{\iota} \| M_{w}\right)+ \\
& +\sum_{x \in \mathbf{D}^{\mathrm{v}}} r 5(x) \cdot\left(K\|D\| T\left\|F_{i}\right\|\left(s 5(P L) R_{x}\right) \| M_{w}\right)+ \\
& +r 5(S R)_{-}\left(K\|D\| T\left\|F_{i}\right\|\left(s 5(P W) P_{\epsilon}\right) \| M_{w}\right)+ \\
& +r 5(B R) \cdot\left(K\|D\| T\left\|F_{i}\right\| P_{\epsilon} \| M_{w}\right)+ \\
& +r 3(S R) \cdot\left(K\|D\| T\left\|F_{i}\right\| P_{\iota} \|\left(s 5(S R)(s 2(P E)+r 5(P W) s 2(P W)) M_{w}\right)+\right. \\
& +\operatorname{rl}(S P)_{\cdot}\left(K\|D\| T\left\|F_{i}\right\| P_{\epsilon} \|\left(s 2(N R) M_{w}\right)\right)+ \\
& +\operatorname{rl}(B R)_{-}\left(K\|D\| T\left\|F_{j}\right\| P_{\iota} \|\left(s 5(B R) M_{w}\right)\right)+ \\
& +r \mathrm{l}(L O) \cdot\left(K\|D\| T\left\|F_{i}\right\| P_{\epsilon} \|\left(s 4(L O)\left(s 2(F E) M_{w}+\cdots M_{r}\right)\right)\right)+ \\
& +\delta)(\text { no communications possible })= \\
& =\theta\left(k(B R) \cdot \partial_{H}\left((s \mathrm{l}(B R) K)\|D\| T\left\|F_{i}\right\| P_{\ell} \| M_{w}\right)+\right. \\
& +k(L O) \cdot \partial_{H}\left((s 1(L O) K)\|D\| T\left\|F_{i}\right\| P_{\imath} \| M_{w}\right)+ \\
& +k(S P) \cdot \partial_{H}\left((s 1(S P) K)\|D\| T\left\|F_{i}\right\| P_{\iota} \| M_{w}\right)+ \\
& +t . \partial_{H}\left(K\|D\|(s 3(S R) T)\left\|F_{i}\right\| P_{c} \| M_{w}\right)= \\
& =k(B R)_{0} \theta\left(t \cdot \partial_{H}\left((s 1(B R) K)\|D\|(s 3(S R) T)\left\|F_{i}\right\| P_{\iota} \| M_{w}\right)+\right. \\
& +(s 1(B R) \mid r 1(B R)) \cdot \partial_{H}\left(K\|D\| T\left\|F_{i}\right\| P_{\iota} \|\left(s 5(B R) M_{w}\right)\right)
\end{aligned}
$$




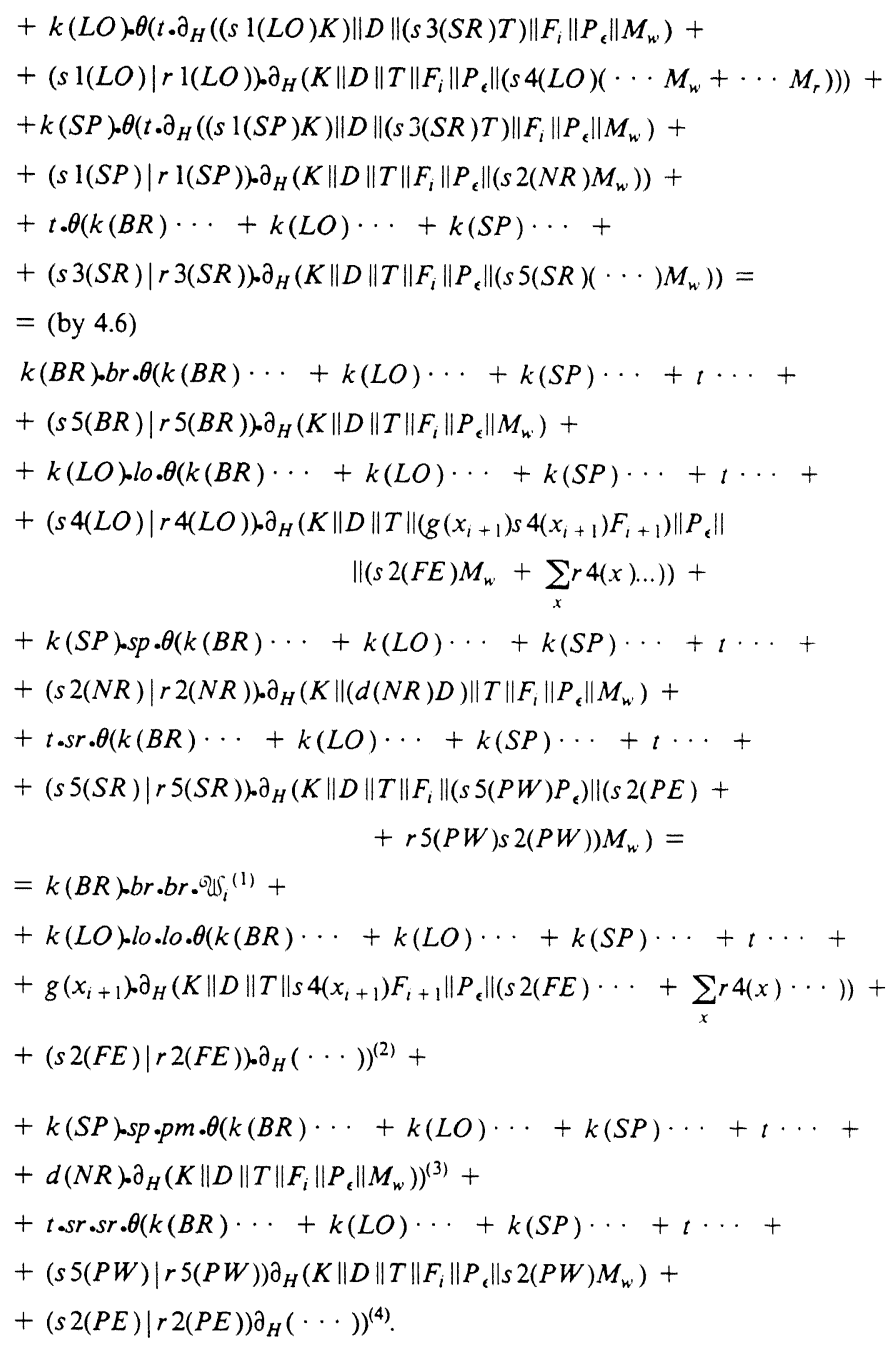

Now term 1 is right, and it is easy to see that term 3 is also right. Therefore, we only continue with terms 2 and 4.

$$
\begin{aligned}
& 2=k(L O) \cdot \operatorname{lo} \cdot \operatorname{lo} \cdot g\left(x_{i+1}\right) \cdot \theta(k(B R) \cdots+k(L O) \cdots+k(S P) \cdots+t \cdots+ \\
& +\left(s 4\left(x_{i+1}\right) \mid r 4\left(x_{i+1}\right)\right) \cdot \partial_{H}\left(K\|D\| T\left\|F_{i+1}\right\| P_{\epsilon} \| s 5\left(x_{i+1}\right)(\cdots)\right)+ \\
& \left.+(s 2(F E) \mid r 2(F E)) \cdot \partial_{H}(\cdots)\right)= \\
& =k(L O) \cdot l o \cdot l o \cdot g\left(x_{i+1}\right) \cdot s d\left(x_{i+1}\right) \cdot \theta(k(B R) \cdots+k(L O) . .+k(S P) \cdots+t \cdots+ \\
& +\left(s 5\left(x_{i+1}\right) \mid r 5\left(x_{i+1}\right)\right) \cdot \partial_{H}\left(K\|D\| T\left\|F_{i+1}\right\| s 5(P L) R_{x_{i+1}+1} \|\right. \\
& \left.\quad \|\left(s 2(P E) M_{w}+r 5(P L) s 2(P L) M_{r}\right)\right)= \\
& +k(L O) \cdot l o \cdot l o \cdot g\left(x_{i+1}\right) \cdot s d\left(x_{i+1}\right) \cdot s d\left(x_{i+1}\right) \cdot \theta(\cdots+\ldots+\cdots+\cdots+ \\
& +(s 5(P L) \mid r 5(P L)) \cdot \partial_{H}\left(K\|D\| T\left\|F_{i+1}\right\| R_{x_{i+1}} \| s 2(P L) M_{r}\right)+
\end{aligned}
$$




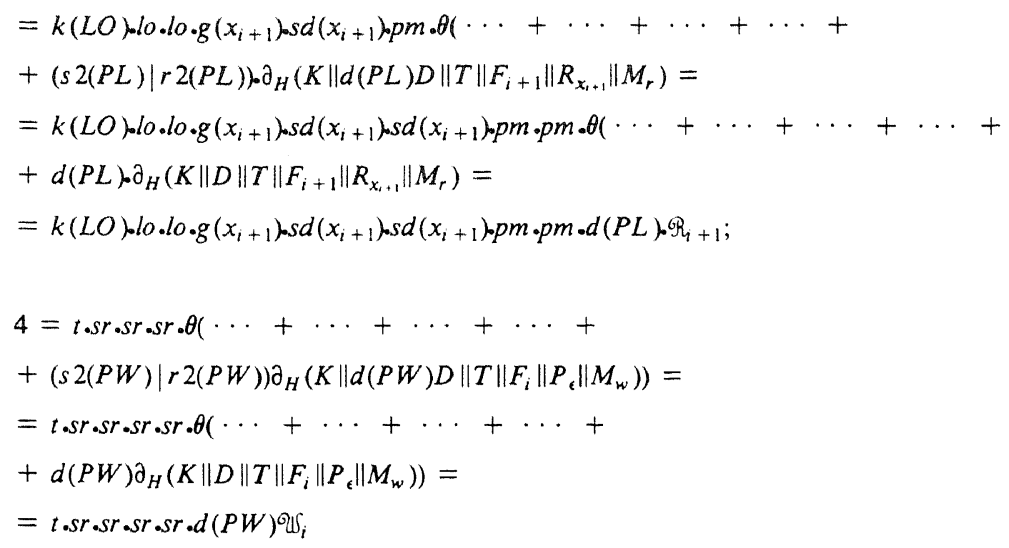

This finishes the proof of 1 .

2. Let $i \geqslant 1$

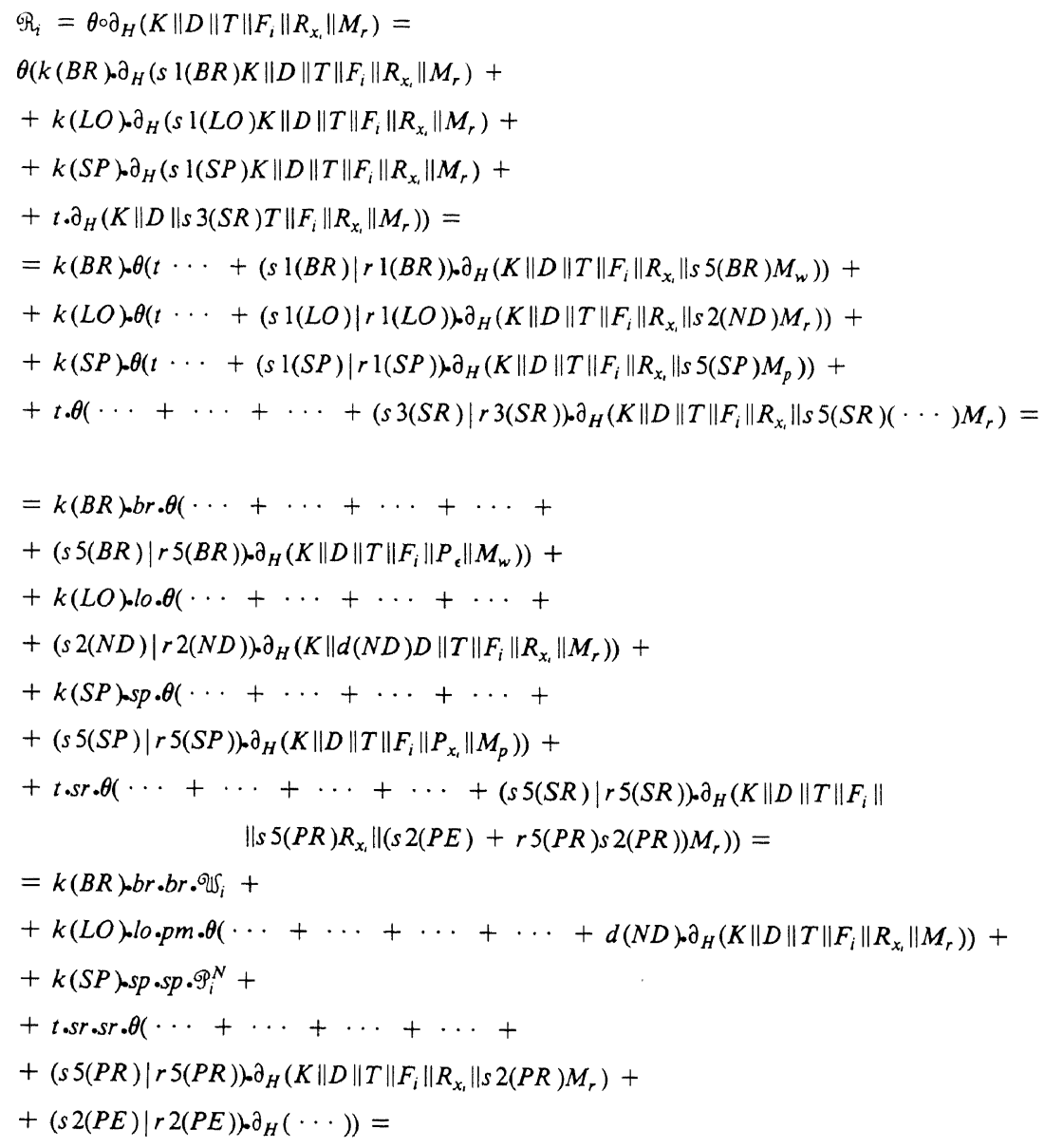




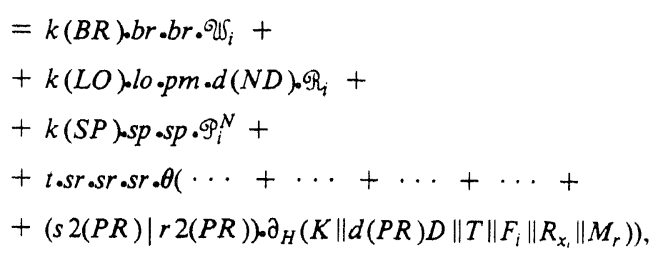

and we can finish as in 1.

3. Let $i \geqslant 1, n=1, \ldots, N$ and $x=d_{1} \cdots d_{n}$ is (the tail of) $x_{i}$. Then $d_{1}$ is the $(N+1-n)^{t h}$ character of $x_{i}$.

case 1: $n>1$. Then

$\mathscr{P}_{i}^{n}=\theta \circ \partial_{H}\left(K\|D\| T\left\|F_{i}\right\| P_{x} \| M_{p}\right)=$

$=\theta\left(k(B R) \cdot \partial_{H}\left(s 1(B R) K\|D\| T\left\|F_{i}\right\| P_{x} \| M_{p}\right)+\right.$

$+k(L O) \cdot \partial_{H}\left(s 1(L O) K\|D\| T\left\|F_{i}\right\| P_{x} \| M_{p}\right)+$

$+k(S P) \cdot \partial_{H}\left(s 1(S P) K\|D\| T\left\|F_{i}\right\| P_{x} \| M_{P}\right)+$

$+t \cdot \partial_{H}\left(K\|D\| s 3(S R) T\left\|F_{i}\right\| P_{x} \| M_{p}\right)+$

$\left.+p\left(d_{1}\right) \partial_{H}\left(K\|D\| T\left\|F_{i}\right\| P_{d_{2}} \ldots d_{n} \| M_{p}\right)\right)$

$=k(B R)(s 1(B R) \mid r 1(B R)), \theta \circ \partial_{H}\left(K\|D\| T\left\|F_{i}\right\| P_{x} \| s 5(B R) M_{w}\right)+$

$+k(L O)(s 1(L O) \mid r 1(L O)) \cdot \theta \circ \partial_{H}\left(K\|D\| T\left\|F_{i}\right\| P_{x} \| s 2(N D) M_{p}\right)+$

$+k(S P)(s 1(S P) \mid r 1(S P)) \cdot \theta \circ \partial_{H}\left(K\|D\| T\left\|F_{i}\right\| P_{x} \| M_{p}\right)+$

$+t(s 3(S R) \mid r 3(S R)), \theta \circ \partial_{H}\left(K\|D\| T\left\|F_{i}\right\| P_{x} \| s 5(S R) \cdots M_{p}\right)$

$+p\left(d_{1}\right) \mathscr{P}_{i}^{n-1}$

Again it is easy to finish the proof.

case 2: $n=1$. The first four terms are the same, so

$\mathscr{P}_{i}^{1}=\theta \circ \partial_{H}\left(K\|D\| T\left\|F_{i}\right\| P_{d_{1}} \| M_{p}\right)=$

$=k(B R) \cdot b r \cdot b r \cdot W_{i}+k(L O) \cdot l o \cdot p m \cdot d(N D) \mathscr{P}_{i}^{l}+$

$+k(S P)_{\text {sp }} \cdot \mathscr{P}_{i}^{1}+t . s r . s r . s r . s r . d(P P) \mathscr{P}_{i}^{1}+$

$+p\left(d_{1}\right) \theta_{\circ} \partial_{H}\left(K\|D\| T\left\|F_{i}\right\| s 5(P D) P_{e} \| M_{p}\right) \quad$ (by 4.3.5.iv) $=$

$=\cdots+\cdots+\cdots+\cdots+$

$+p\left(d_{1}\right) \mathscr{P}_{i}^{0}$

4. Let $i \geqslant 1$.

$\mathscr{P}_{i}^{0}=\theta \circ \partial_{H}\left(K\|D\| T\left\|F_{i}\right\| s 5(P D) P \| M_{p}\right)=$

$=\theta(k(B R) \cdots+k(L O) \cdots+k(S P) \cdots+t \cdots+$

$\left.+(s 5(P D) \mid r 5(P D)) \cdot \partial_{H}\left(K\|D\| T\left\|F_{i}\right\| P \| s 2(P D) M_{w}\right)\right)=$

$=p m \cdot \theta(\cdots+\cdots+\cdots+\cdots+$

$\left.+(s 2(P D) \mid r 2(P D)) \cdot \partial_{H}\left(K\|d(P D) D\| T\left\|F_{i}\right\| P \| M_{w}\right)\right)=$

$=p m \cdot p m \cdot \theta(\cdots+\cdots+\cdots+\cdots+$

$\left.+d(P D) \cdot \partial_{H}\left(K\|D\| T\left\|F_{i}\right\| P \| M\right)\right)=$

$=p m \cdot p m \cdot d(P D) \cdot w_{i}$. 
This completes the proof of theorem 4.8 .

4.9 Now we want to focus on certain aspects of the system just described, and abstract from others. We will present three ways of doing this, namely:

1. abstract from all internal steps, focus on key-board, display and printer;

2. abstract from all internal steps, focus on file and printer (i/o view);

3. get easier equations for 2 by hiding interrupts.

As in 3.8, we work in $\mathrm{ACP}_{\tau}$, but now we need an extra abstraction rule, since infinite $\tau$-paths can occur. The rule is explained in 4.10 .

4.10 Koomen's fair abstraction rule (KFAR) (see Bergstra \& Klop [3]).

This rule allows us to compute $\tau_{I}(X)$ for certain $X$, thereby expressing the fact that certain steps in I will be fairly scheduled in such a way that eventually a step outside $I$ is performed. Formally,

$$
(\mathrm{KFAR}) \frac{\forall n \in \mathbb{Z}_{k} X_{n}=i_{n} \cdot X_{n+1}+Y_{n}\left(i_{n} \in I\right)}{\tau_{I}\left(X_{n}\right)=\tau \cdot \tau_{I}\left(Y_{0}+\cdots+Y_{k-1}\right)}
$$

Here $\mathbb{Z}_{k}=\{0, \ldots, k-1\}$ and addition in subscripts works modulo $k$. For the use of KFAR, also see [3].

$$
\begin{aligned}
& \text { 4.11 Define } I=\{t, b r, s r, l o, s p, p m, e m\} \cup \\
& \qquad\left\{g(x) \mid x \in \mathbb{D}^{N}\right\} \cup\left\{s d(x) \mid x \in \mathbb{D}^{N}\right\} \cup \\
& \cup\{d(P W), d(P R), d(P P)\} .
\end{aligned}
$$

This means we abstract from the timer, the file, all communications and the status reports. Now applying $\tau_{I}$ to the equations of theorem 4.8 , and using KFAR, gives:

1. $\tau_{I}\left(\varpi_{i}\right)=\tau\left((k(B R)+k(S P) d(N R)) \tau_{I}\left(\mathscr{W}_{i}\right)+\right.$

$$
\left.+k(L O) d(P L) \tau_{I}\left(\Re_{i+1}\right)\right) \quad(i \geqslant 0)
$$

2. $\tau_{I}\left(\Re_{i}\right)=\tau\left(k(B R) \tau_{I}\left(\mho_{i}\right)+\right.$

$$
\begin{aligned}
& +k(L O) d(N D) \tau_{I}\left(\Re_{i}\right)+ \\
& \left.+k(S P) \tau_{I}\left(\mathscr{P}_{i}^{N}\right)\right) \quad(i \geqslant 1)
\end{aligned}
$$

3. $\tau_{I}\left(\oplus_{i}^{n}\right)=\tau\left(k(B R) \tau_{I}\left(\mho_{i}\right)+\right.$

$$
+k(L O) d(N D) \tau_{I}\left(\mathscr{P}_{i}^{n}\right)+
$$$$
+k(S P) \tau_{I}\left(\mathscr{P}_{i}^{n}\right)+
$$$$
\left.+p(d) \oplus_{i}^{n-1}\right) \quad(i \geqslant 0, n=1, \ldots, N \text {, }
$$$$
\left.d \text { is the }(N+1-n) \text { th character of } x_{i}\right)
$$

4. $\quad \tau_{I}\left(\mathscr{P}_{i}^{0}\right)=\tau d(P D) \tau_{I}\left(\mho_{i}\right)$.

We can make the following state transition diagram of $\tau_{I}\left(W_{0}\right)$. (Suppose $x_{1}=d_{1} \cdots d_{N}$ ) 


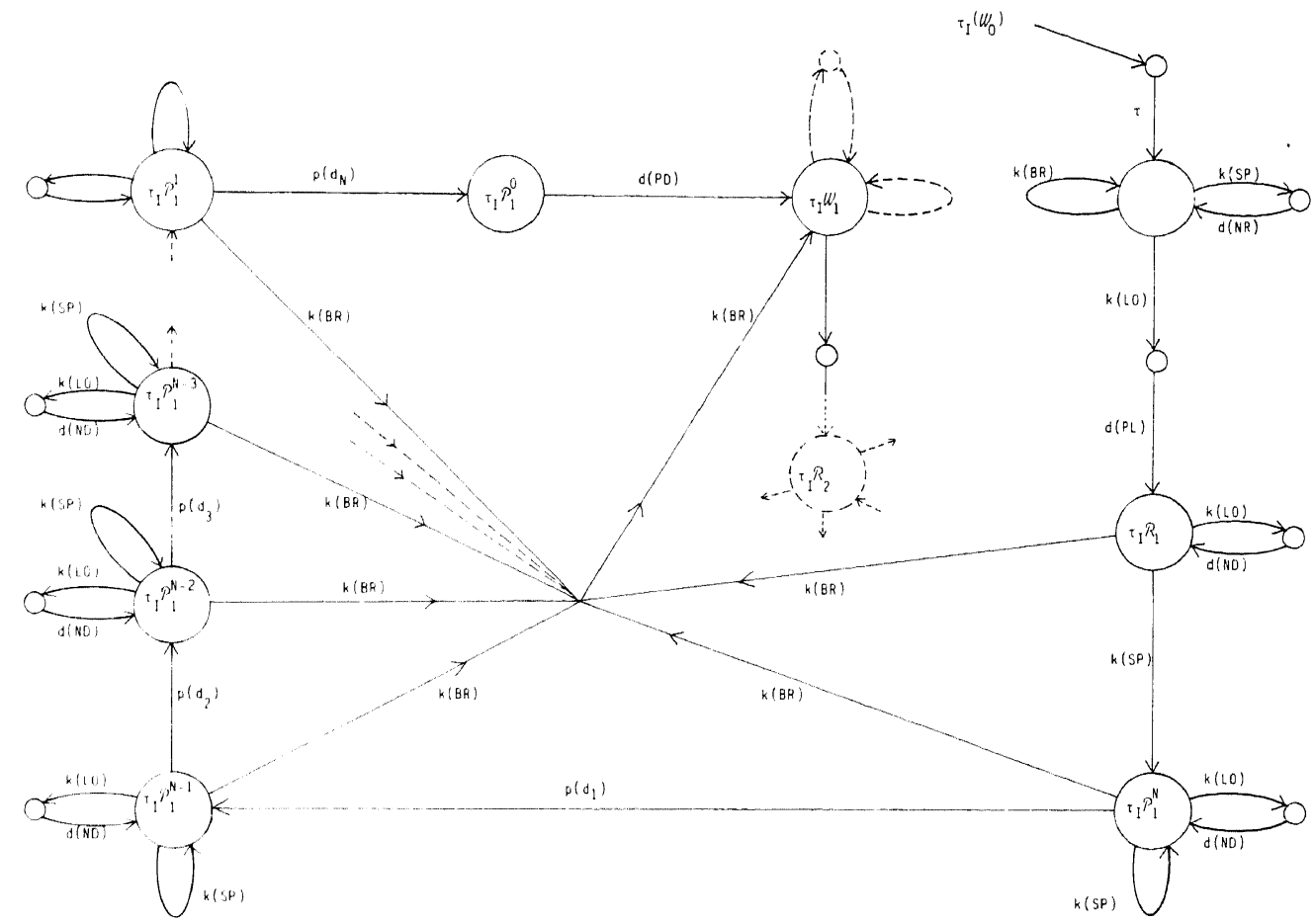

4.12 Second version:

fig. 38

Diagram:

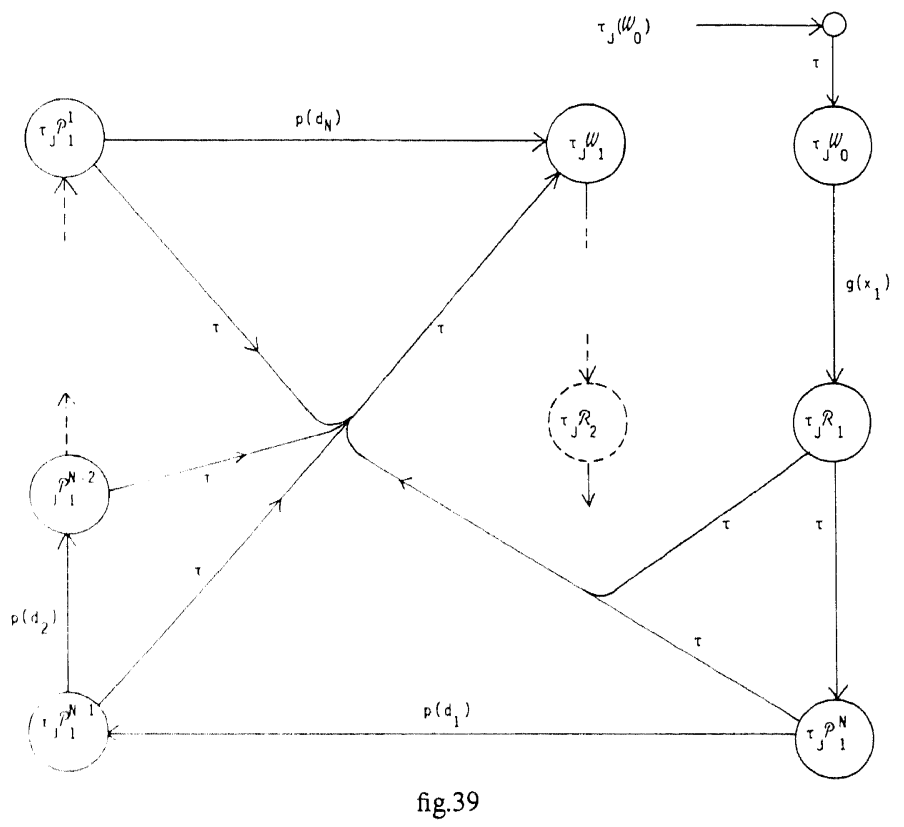




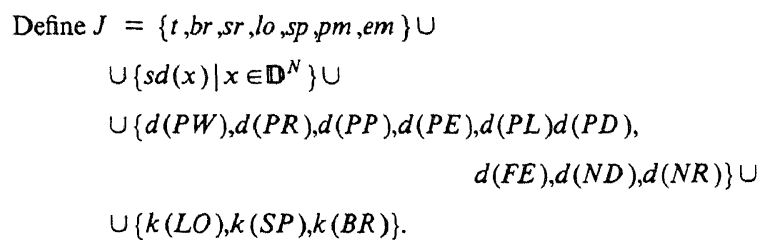

This means we abstract from the timer, the display, the keyboard and all communications. Now apply $\tau_{J}$ and use KFAR. We get:

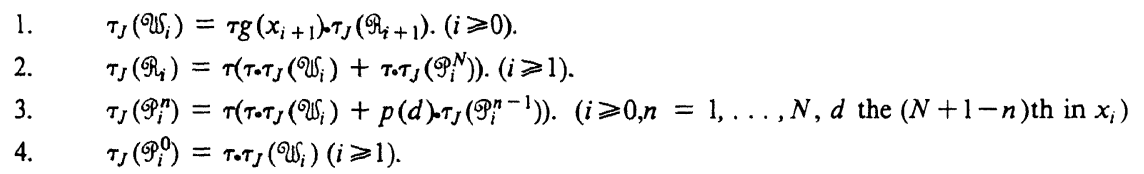

4.13 In order to see better, that the input is really that what is printed, we will use the following trick. Define $B=\{k(B R)\}$ and look at

$$
\tau_{J} \circ \partial_{B}\left(\text { ข }_{i}\right)
$$

This means that we throw away all paths that have a break, and then do the abstraction of 3.12

Claim: Suppose $x_{i+1}=d_{1}^{i} \cdots d_{N}^{i},(i \geqslant 0)$.

$$
\tau_{J} \circ \partial_{B}\left(\mho_{i}\right)=\tau \cdot g\left(x_{i+1}\right) p\left(d_{1}^{i}\right) p\left(d_{2}^{i}\right) \cdots p\left(d_{N}^{i}\right) \circ \tau_{J}^{\circ} \partial_{B}\left(\mho_{i+1}\right) .
$$

Proof: we start with the equations of theorem 3.8.

$$
\begin{aligned}
\partial_{B}\left(\mho_{i}\right)=\delta & +a_{1} a_{2} a_{2} \cdot g\left(x_{i+1}\right) a_{3} a_{3} a_{4} a_{4} a_{5} \partial_{B}\left(\mathscr{R}_{i+1}\right)+ \\
& +a_{6} a_{7} a_{4} a_{8} \partial_{B}\left(\mho_{i}\right)+a_{9} a_{10} a_{10} a_{10} a_{10} a_{11} \partial_{B}\left(\mho_{i}\right)
\end{aligned}
$$

(here the $a_{1}-a_{11}$ symbolize internal steps), so by KFAR we have

1. $\tau_{J} \circ \partial_{B}\left(\mho_{i}\right)=\tau g\left(x_{i+1}\right) \circ \tau_{J}^{\circ} \partial_{B}\left(\Re_{i+1}\right)$.

2. $\tau_{J} \circ \partial_{B}\left(\Re_{i+1}\right)=\tau \cdot \tau_{J} \circ \partial_{B}\left(\mathscr{P}_{i+1}^{N}\right)$

3. $\tau_{J} \circ \partial_{B}\left(\Im_{i+1}^{n}\right)=\tau p\left(d_{N+1-n}^{i}\right) \cdot \tau_{J} \circ \partial_{B}\left(\oplus_{i+1}^{n-1}\right)(n=1, \ldots, N)$

$$
\text { and }
$$

4. $\tau_{J} 0 \partial_{B}\left(\oplus_{i+1}^{\circ}\right)=\tau \cdot \tau_{J} \circ \partial_{B}\left(\mathcal{W}_{i+1}\right)$.

Combining these gives

$$
\begin{aligned}
& \tau_{J} \circ \partial_{B}\left(\mho_{i}\right)=\tau g\left(x_{i+1}\right) \circ \tau_{J} \circ \partial_{B}\left(\Re_{i+1}\right)= \\
& =\tau g\left(x_{i+1}\right) \cdot \tau \cdot \tau_{J} \circ \partial_{B}\left(\mathscr{\Phi}_{i+1}^{N}\right)= \\
& =\tau g\left(x_{i+1}\right) \cdot \tau_{\cdot} p\left(d_{1}^{i}\right) \circ \tau_{J}^{\circ} \circ \partial_{B}\left(\mathscr{P}_{i+1}^{N-1}\right)= \\
& =\tau g\left(x_{i+1}\right) \cdot p\left(d_{1}^{i}\right) \cdot \tau_{\cdot} p\left(d_{2}^{i}\right) \cdot \tau_{j} \circ \partial_{B}\left(\mathscr{P}_{i+1}^{N}-2\right)= \\
& =\ldots . .= \\
& =\tau g\left(x_{i+1}\right) p p\left(d_{1}^{i}\right) p\left(d_{2}^{i}\right) \cdots \cdot \tau p\left(d_{N}^{i}\right) \cdot \tau_{J} \circ \partial_{B}\left(\mathscr{P}_{i+1}^{0}\right)= \\
& =\tau g\left(x_{i+1}\right) \cdot p\left(d_{1}^{i}\right) p\left(d_{2}^{i}\right) \cdots p\left(d_{N}^{i}\right) \tau \cdot \tau_{J} \circ \partial_{B}\left(\mho_{i+1}\right)= \\
& =\tau g\left(x_{i+1}\right) \cdot p\left(d_{1}^{i}\right) p\left(d_{2}^{i}\right) \cdots p\left(d_{N}^{i}\right) \tau_{J} \circ \partial_{B}\left(\mho_{i+1}\right) \text {. }
\end{aligned}
$$




\section{References}

[1] De BAKkeR, J.W. \& J.I. ZUCKer, Processes and the denotational semantics of concurrency, Information and Control, 54 (1/2), 1982, pp.70-120.

[2] Bergstra, J.A. \& J.W. Klop, Algebra of communicating processes with abstraction, Theoretical Comp. Sci. 37 (1), 1985, pp. 77-121.

[3] BERGSTRA, J.A. \& J.W. KLOP, Verification of an alternating bit protocol by means of process algebra, Report CS-R8404, Centrum voor Wiskunde en Informatica, Amsterdam 1984.

[4] Bergstra, J.A. \& J.W. Kıop, Process algebra for synchronous communication, Information and Control $60(1 / 3), 1984$, pp. 109-137.

[S] Bergstra, J.A. \& J.V. TUCKER, Top-down design and the algebra of communicating processes, Science of Comp. Programming 5 (2), 1985, pp. 171-199.

[6] DeRshowitz, N., Orderings for term-rewriting systems, Theoretical Computer Science 17, 1982, pp. 279-301.

[7] HUET, G., Confluent reductions: abstract properties and applications to term rewriting systems, J. ACM 27 (4), 1980, pp. 797-821.

[8] Kıop, J.W., Combinatory reduction systems, Mathematical Centre Tract 127, Mathematisch Centrum, Amsterdam 1980.

[9] MILNER, R., A calculus for communicating systems, Springer LNCS 92, 1980.

[10] PARK, D.M.R., Concurrency and automata on infinite sequences, Springer LNCS 104, 1981. 